\title{
Temporal control of cortico-thalamic neuron specification by regulation of Neurogenin activity and Polycomb repressive complexes
}

Koji Oishi ${ }^{1, *}$, Debbie L. C. van den Berg, François Guillemot ${ }^{2, *}$

The Francis Crick Institute, 1 Midland Road, London NW1 1AT, U.K.

${ }^{1}$ Present address: Department of Anatomy, Keio University School of Medicine, 35 Shinanomachi, Shinjuku-ku, Tokyo 160-8582, Japan

${ }^{2}$ Lead Contact

*Correspondence: k-oishi@keio.jp (K.O.) or francois.guillemot@crick.ac.uk (F.G.)

Key words: Neocortex; neurogenesis; fate specification; Neurogenin; Polycomb 


\section{Summary}

Neural progenitor cells (NPCs) in the embryonic mammalian neocortex generate different neuronal subtypes sequentially. A long-standing hypothesis to account for this temporal fate specification process is that NPCs change their differentiation potential over time. However, the molecular mechanisms underlying these temporal changes in NPC properties are poorly understood. Here we show that Neurogenin1 and Neurogenin2 (Neurog1/2), two proneural transcription factors expressed in NPCs throughout cortical neurogenesis, specify the identity of one of the first cortical neuron subtypes generated, layer 6 cortico-thalamic neurons (CTNs). We found that Neurog1/2 specify the CTN fate through regulation of the cortical fate determinants Fezf 2 and Foxp 2 and that this Neurog-induced programme becomes inactive after the period of CTN production. Two independent mechanisms contribute to the arrest of CTN neuron generation at the end of layer 6 neurogenesis, including a reduction in the transcriptional activity of Neurog $1 / 2$ and the deposition of epigenetic repressive modifications mediated by Polycomb repressive complexes at the Foxp2 gene. Therefore, the duration of production of a cortical neuron subtype is controlled by multiple locking mechanisms involving both transcriptional and epigenetic processes. 


\section{Introduction}

Neural stem cells and intermediate progenitors (called thereafter neural progenitor cells or NPCs) which reside in the ventricular and subventricular zones (VZ/SVZ) of the mammalian neocortex, give rise to distinct subtypes of neurons with specific cell morphologies, birth dates and connections with other regions of the nervous system (Molyneaux et al., 2007; Lodato and Arlotta, 2015; Martynoga et al., 2012). Cortical neurons are born either directly from stem cells in the VZ or indirectly from intermediate progenitors which reside in the cortical SVZ (Florio and Huttner, 2014; Sun and Hevner, 2014). They are generated in a sequential manner, with the earliest NPCs generating subplate neurons around E11.5 while subsequent NPCs produce neurons in layers 6, 5, 4, and eventually $2 / 3$ around E12.5, E13.5, E14.5 and E15.5, respectively. This crucial feature of cortical development has led to a model whereby cortical neurons acquire their distinct laminar identities by temporal specification of cortical NPCs, with the differentiation potential of NPCs changing over time (Greig et al., 2013; McConnell, 1995), akin to the temporal specification of neuroblasts in the ventral nerve cord and optic lobe of Drosophila (Kohwi and Doe, 2013). However, there is scant evidence that NPCs are temporally specified during mammalian corticogenesis and few temporally-controlled factors that might drive such a process have been identified (Alsio et al., 2013; Dominguez et al., 2013; Frantz et al., 1994; Hirata et al., 2004; Zahr et al., 2018).

Studies from Sue McConnell's group involving heterochronic transplantation experiments have demonstrated that cortical NPCs undergo a progressive restriction of fate potential during cortical development. They showed that early NPCs, producing layer 6 neurons, could differentiate into upper layer neurons when transplanted in the late VZ, while late NPCs, producing layer 2/3 neurons did not differentiate into deep layer neurons when transplanted into 
the early VZ (Frantz and McConnell, 1996; McConnell and Kaznowski, 1991). Recent studies have identified molecular determinants that are critical for the specification of particular cortical neuron subtypes, but most of these factors are expressed only in postmitotic neurons [e.g. Bcl11b (Arlotta et al., 2005); Bhlhb5 (Joshi et al., 2008); Rorb (Oishi et al., 2016a); Pcdh20 (Oishi et al., 2016b); Zfpm2 (Galazo et al., 2016)] or in both NPCs and neurons [Fezf2 (Hirata et al., 2004; Molyneaux et al., 2005), Pou3fs (Dominguez et al., 2013), Otx1 (Frantz et al., 1994)]. It remains therefore unclear whether cell fate determination actually occurs in NPCs as posited by the temporal specification hypothesis (Alsio et al., 2013).

We previously reported that Neurog1/2, two proneural basic helix-loop-helix (bHLH) transcription factors that are expressed in NPCs and only transiently in postmitotic neurons, promote the generation and neuronal differentiation of cortical neurons but also the acquisition of aspects of the subtype identity of deep layer (early born) neurons, and not that of upper layer (late born) neurons (Schuurmans et al., 2004). However, the downstream mechanisms through which Neurogl/2 specify a deep layer fate have not yet been determined. Moreover, the fact that Neurog1/2 are prominently expressed by NPCs throughout the neurogenic period although they specify neuronal subtypes only during the early stages of neurogenesis, raises the question of how this fate specification mechanism is temporally regulated.

Epigenetic control of gene expression, and particularly chromatin remodeling by modifications of histones, have been shown to play pivotal roles in cell fate specification in a wide variety of cell types including NPCs (Grossniklaus and Paro, 2014; Margueron and Reinberg, 2011; Yoon et al., 2018). Polycomb repressive complexes (PRCs) in particular have been implicated in the regulation of cerebral cortex development, including in the timing of generation of particular cortical cell types. PRCs are composed of PRC2, which deposits methyl 
residues on Lys27 in Histone H3 (H3K27me3) and PRC1, which recognizes H3K27me3 and ubiquitinates Lys119 in Histone H2A (H2AK119ub) (Blackledge et al., 2015; Simon and Kingston, 2013). Ezh2, a catalytic component of PRC2, has been reported to play a role in the balance between self-renewal and differentiation of cortical NPCs (Pereira et al., 2010). Moreover, in the absence of Ezh2, many subtype-specific markers are expressed prematurely in the early cortex (Pereira et al., 2010), suggesting a global repression of subtype specific genes by PRCs. More recently, Ring1B, a catalytic subunit of PRC1, has been implicated in the restriction of the differentiation potential of cortical NPCs. Ring $1 B$ knockout was reported to extend the period of generation of layer 5 neurons and to reduce the period of generation of upper layer neurons, suggesting that PRC1 is required for the termination of layer 5 production (Morimoto-Suzki et al., 2014). How widespread is the role of PRCs in the temporal specification of cortical neuron subtypes is however still unclear and whether they control the timing of Neurog $1 / 2$ activity of deep layer neuron specification is unknown.

In this study, we have further defined the role of Neurogl/2 in specification of deep layer cortical neurons and we have identified the mechanisms that restrict temporally this activity without affecting Neurogl/2 proneural function that operates throughout cortical development. We found that changes in both Neurog1/2 transcriptional activity and PRCdependent regulation of some of their targets restrict Neurog $1 / 2$ subtype specification function during cortical development. 


\section{Results}

\section{Failure of generation of cortico-thalamic neurons in Neurogenin mutant mice}

Our previous work showed that Neurogl/2 are required for the correct specification of several aspects of deep layer cortical neuron identities but not for the specification of superficial layer neuronal identities (Schuurmans et al., 2004). However, because of a lack of appropriate markers at the time, it was unclear which particular subtypes of deep layer neurons, which include layer 5 subcerebral (e.g. cortico-spinal, cortico-pontine) neurons, layer 6 corticothalamic neurons (CTNs) and layer 6 and layer 5 cortico-cortical (CCNs) neurons, require Neurog1/2 for their specification. To better define the role of Neurogl/2 in cortical neuron subtype specification, we re-examined the neocortex of Neurog $1 / 2$ mutant mice using markers specific for each of the different subtypes of deep layer neurons.

Bcl11b (also known as Ctip2), a well-established marker for subcortical neurons, is expressed strongly by layer 5 subcerebral neurons and at a lower level by layer 6 CTNs (Arlotta et al., 2005). We observed a decrease (46\% decrease) in the number of Bcl11b-positive cells in layer 6, corresponding to CTNs, but not in layer 5, corresponding to subcerebral neurons, in Neurog2 mutant mice and more strongly in Neurog $1 / 2$ mutants (64\% decrease) at E18.5 (Figures 1A-1C and 1M). Moreover, the number of Satb2-positive cells in layer 5, corresponding to layer $5 \mathrm{CCNs}$, remained unchanged in Neurog2 and Neurog $1 / 2$ mutants (Figures 1A-1C and $1 \mathrm{~N}$ ). This finding prompted us to investigate in detail the specification of layer 6 neurons in these mice. We first examined whether the entire layer 6 neuronal population was affected in Neurog1/2 mutants. The number of cells in layer 6, visualized by nuclear staining, was slightly reduced in Neurog2 and Neurog $1 / 2$ mutants (16\% and 17\% decrease, respectively) (Figure 1O). However, the extent of reduction of Bcl11b-positive cells in layer 6 
was more profound than that of all layer 6 neurons (46\% versus $16 \%$ decrease), and the percentage of Bcl11b-positive cells among layer 6 neurons was decreased in Neurog2 and Neurog $1 / 2$ mutants (36\% and 56\% decrease, respectively) (Figure 1P).

As Bcl11b was reported to label CTNs, we also examined the expression of other CTN makers, including the transcription factors Foxp2 (Hisaoka et al., 2010), Zfpm2 (Han et al., 2011), Nfia (Betancourt et al., 2014) and the signal transduction protein Ppp1r1b/Darpp32 (Molyneaux et al., 2005). The numbers of cells expressing these markers were all reduced in Neurog2 and Neurog1/2 mutant mice (Figures 1D-1I, 1Q and 1R; Figures S1A-S1F and S1M), confirming that Neurog 1 and Neurog2 are required for the generation of CTN neurons.

\section{Fate switch of Neurogenin-deficient cortico-thalamic neurons to cortico-cortical neurons}

The observed reduction in percentage of CTNs among layer 6 neurons in Neurog mutant mice suggested that some CTNs might be mis-specified and acquire alternative identities in the absence of Neurog1/2. CCNs, which send axons to the contralateral or ipsilateral cortex, constitute another major subtype of layer 6 neurons (Thomson, 2010; Tasic et al., 2016), which can be distinguished from CTNs by the expression of Satb2 and Nurr1 (Arimatsu et al., 2003; Watakabe et al., 2007). The numbers of Satb2- and Nurr1-positive cells in layer 6 was indeed increased in E18.5 Neurog2 and Neurong1/2 mutant mice (Satb2, 31\% and 51\%, respectively; Nurr1, 46\% and 77\%, respectively) (Figures $1 \mathrm{~A}-1 \mathrm{C}$ and $1 \mathrm{~N}$; Figures S1G-S1I and S1N). Importantly, all cells located in layer 6 of Neurog mutant cortices retained molecular characteristics of layer 6 neurons, since they expressed Tbr1, a pan-layer 6 marker (Figures $1 \mathrm{~J}-$ 1L; (Galazo et al., 2016)) and Sox5, a maker of all layer 6 and a subset of layer 5 neurons (Figures S1J-S1L). Therefore, a large fraction of layer 6 neurons fail to be specified into CTNs 
and switch to another layer 6 subtype, CCNs, in the absence of Neurog $1 / 2$ function.

\section{Neurog2 overexpression promotes cortico-thalamic neuron specification}

We next asked whether Neurog $1 / 2$ expression was not only required but also sufficient to induce a CTN fate in NPCs destined to produce layer 6 neurons. To address this question, we used in utero electroporation (IUE) to express Neurog2 and the reporter EGFP in cortical VZ cells at around E12.5, when most layer 6 neurons are born (Figure 2A). In a control experiment where EGFP alone was electroporated, $82.2 \pm 1.8 \%$ of EGFP labeled cells analysed at E18.5 were located in layer 6 and $40-50 \%$ of the electroporated cells co-expressed CTN markers including Foxp2 (Figures 2A and 2G), Zfpm2 (Figure 2C and 2H), Bcl11b (Figures S2A and S2E) and Nfia (Figures S2C and S2F), while $48.6 \pm 1.8 \%$ expressed the CCN marker Satb2 (Figures 2E and 2I). Co-expression of Neurog2 with EGFP markedly increased the percentages of CTN marker-positive cells to 75-90\% (Figures 2B, 2D, 2G, and 2H; Figures S2B and S2D-S2F), while decreasing the percentage of Satb2-positive CCNs to $14.4 \pm 3.4 \%$ (Figures $2 \mathrm{~F}$ and 2I). Importantly, the axons of neurons electroporated with Neurog $2+E G F P$ were more abundant in the dorsal thalamus, corresponding to the trajectory of CTN axons, than those from control neurons (Figures $2 \mathrm{~J}-2 \mathrm{~N}$ ), suggesting that Neurog2 overexpression changed not only marker gene expression but also the axonal projection pattern of electroporated neurons to a CTN-like phenotype.

Neurog overexpression has been shown to promote cell cycle exit and neuronal differentiation (Hand et al., 2005; Nieto et al., 2001). The Neurog2-induced conversion of CCNs into CTNs could in principle be due to the premature differentiation of Neurog2electroporated neurons if CTNs were normally generated before CCNs. To exclude this 
possibility, we compared Neurog2-electroporated and control EGFP-electroporated neurons that were born at the same time by administering EdU one hour before IUE and focusing our analysis on the population of EGFP+ cells that were strongly labeled with EdU, i.e. cells that were NPCs in S phase of the cell cycle at the time of IUE and became postmitotic immediately thereafter (Figure 2O). Neurog2 overexpression in this population markedly increased the percentage of Zfpm2-positive CTNs compared with the percentage of Zfpm2-expressing CTNs born at the same time in the control electroporation experiment (Figures 2P-2R), suggesting that Neurog2 directly promotes the CTN fate rather than controlling the timing of layer 6 neuron differentiation. Therefore, expression of Neurogenins during the generation of layer 6 neurons is sufficient to promote $\mathrm{CTN}$ specification at the expense of $\mathrm{CCN}$ specification.

\section{Identification of target genes of Neurog 2 involved in cortico-thalamic neuron specification}

Neurogenin genes specify CTNs presumably by inducing CTN-specific target genes in NPCs.

To identify such targets, we mined multiple datasets, including microarray data for genes expressed in Neurog2 mutant, Neurog1/2 mutant and control E13.5 cortices (Gohlke et al., 2008), ChIP-Seq data for genomic locations bound by Neurog2 in E14.5 cortex (Sessa et al., 2016), publicly available in situ hybridization resources including the Allen Developing Mouse Brain Atlas (http://developingmouse.brain-map.org) and BGEM (Magdaleno et al., 2006), and literature searches for genes expressed in mouse cortical layer 6 neurons. Since Neurog $1 / 2$ are expressed in NPCs, we speculated that candidate targets that can execute CTN specification in neurons should include transcription factors that are expressed in NPCs and/or postmitotic neurons. Using these datasets, we identified several relevant candidate Neurog target genes, i.e. genes encoding transcription factors that are down-regulated in Neurog2 and Neurog1/2 mutant 
cortices and have Neurog2 ChIP-Seq peaks in their vicinity (Figure 3A). We narrowed further the candidate targets list by examining the effect of Neurog2 overexpression, which is expected to increase the expression of bona fide targets since Neurog2 has been shown to act as a transcriptional activator (Castro et al., 2006). EGFP-positive cells from cortices electroporated with Neurog2 plus EGFP or EGFP alone at E12.5 and dissociated at E16.5 were purified by FACS and the mRNA was prepared for qRT-PCR analysis. RNA levels for some of the candidate targets including Bcl11b, Fezf2, Nfia and Foxp2 were significantly up-regulated by Neurog2 overexpression (Figure 3B).

To provide further evidence for direct regulation by Neurog2, we determined whether the Neurog2 binding sites identified by ChIP-Seq actually mediated Neurog2-dependent transcription. We cloned in a luciferase reporter construct Neurog2-bound regions that are well conserved between mouse and human (ECR browser (Ovcharenko et al., 2004)), since important regulatory elements such as enhancers tend to be conserved between mammalian species (Elnitski et al., 2003; Loots et al., 2000). The conserved, Neurog2-bound genomic regions associated with Fezf2 [48kb upstream of Fezf2 transcription start site (TSS)] and Nfia (345kb upstream of Nfia TSS) induced luciferase activity upon Neurog2 expression in the neural stem cell line NS-5 (Figure 3C). In contrast, Ascl1, another proneural bHLH transcription factor, did not promote transcription in these assays, while both Neurog2 and Ascll activated transcription when interacting with an element in the Foxp2 gene $(0.8 \mathrm{~kb}$ upstream of the Foxp2 TSS) (Figure 3C).

The transcription factor Fezf2 has previously been implicated in the specification of CTNs in layer 6 (Chen et al., 2005a; Molyneaux et al., 2005) and of subcerebral projection neurons in layer 5 (Chen et al., 2005a; Chen et al., 2005b; Molyneaux et al., 2005). Since Fezf2 
expression was reduced in Neurog2 and Neurog1/2 mutant cortices (Figure 3A) and was induced by overexpression of Neurog2 (Figure 3B), and since Neurog2 was bound to a conserved element 48kb upstream of Fezf2 TSS (Figure 3A) and could specifically induce transcription when interacting with this element (Figure 3C), Fezf2 appeared to be a good candidate to mediate some of the activity of Neurog genes in specification of CTNs. We found five consensus binding motifs for bHLH factors (E-boxes) in the Fezf2-48kb genomic sequence that responded to Neurog2 expression in the luciferase assay (Figure 3D). The third motif (E3) matches perfectly the E box CA $\underline{\text { GATG }}$ which is specifically recognized by Neurogenin proteins (Seo et al., 2007) and is present in Neurogenin-regulated enhancers in the Neurod1 and Rnd2 genes (Heng et al., 2008; Huang et al., 2000). A luciferase construct harboring tandem repeats of the E3 motif was strongly activated by Neurog2 (Figure 3E) and point mutations disrupting this E-box abolished the activation of the Fezf2-48kb element by Neurog2 (Figure 3F), thus providing strong evidence that E3 mediates most of the Neurog2-dependent activity of this enhancer.

We next assessed the activity of the $F e z f 2-48 k b$ enhancer in the embryonic cortex and determined the role of Neurog2 in activating this element in vivo. IUE of the Fezf2-48kb luciferase reporter construct in cortical NPCs at E12.5 strongly induced luciferase activity in vivo 1 day later, when most of the electroporated cells were still located in the VZ/SVZ (Figure $3 G)$. The mutation of the E3 motif reduced the activity of the Fezf2-48kb element by $41 \%$ (Figure 3G), and Neurog2 knockdown by co-electroporation of a Neurog2 shRNA construct decreased the activity of the wild-type element by $30 \%$ (Figure $3 \mathrm{H}$ ), demonstrating that endogenous Neurogenin factors contribute to the activity of this enhancer in vivo.

To further investigate whether Fezf2-48kb regulates the Fezf2 gene in the embryonic 
cortex, we generated a mouse line in which this putative enhancer has been disrupted (Fezf248kbenhKO mutation) using the CRISPR/Cas9 system (Cong et al., 2013; Mali et al., 2013; Yang et al., 2013) (see Methods). The mRNA level of Fezf2 in whole cortices of E14.5 mice homozygous for the Fezf248kbenhKO mutation was significantly reduced compared to that in heterozygous controls (Figure 3I). Fezf2 is expressed at much higher levels in subcerebral layer 5 projection neurons than in CTNs (http://developingmouse.brain-map.org and data not shown) and has been reported to be controlled by multiple enhancers (Han et al., 2011; Kwan et al., 2008; McKenna et al., 2011; Shim et al., 2012). The limited reduction of Fezf2 expression in Fezf248kbenhKO mice could thus be due to its remaining expression in layer 5 subcerebral projection neurons. Collectively, these results indicate that Neurog1/2 proteins control Fezf2 expression in CTNs at least in part by interacting with its $-48 \mathrm{~kb}$ enhancer.

\section{Neurog 2 induces $F e z f 2$ to activate the cortico-thalamic neuron specification programme}

We next examined whether Fezf2 acts downstream of Neurogenins for the specification of CTNs. For this, we first showed that $F e z f 2$ overexpression by IUE promotes CTN specification in the same way as Neurog2 does. Electroporation of Fezf2 at E12.5 increased the generation of Foxp2-, Zfpm2-, Bcl11b- and Nfia-expressing CTNs (Figures 3J-3M; Figures S3A-S3D) and decreased the generation of Satb2-positive CCNs (Figures 3N and 3O).

To determine whether Fezf2 mediates the specification of CTNs by Neurog2, we examined the consequence of suppressing Fezf2 expression in Neurog2-overexpressing cells. We used a Fezf2 knockdown construct (Chen et al., 2005b) that efficiently suppresses Fezf2 expression (Figure S3E) and prevents the generation of CTNs when electroporated in E12.5 embryonic cortices, as expected from the known role of Fezf2 in CTN specification (Chen et 
al., 2005a; Molyneaux et al., 2005) (control experiments in Figures 3T and 3U). Coelectroporation of Neurog2 with the Fezf2 knockdown construct failed to induce the generation of CTNs, in contrast to electroporation of Neurog2 alone (Figures 3P-3U; Figures S3F and S3G). Therefore, Fezf2 acts downstream of the Neurogenin genes and is essential for their activity of specification of the CTN fate.

\section{Neurog2 overexpression fails to induce cortico-thalamic neurons after E12.5}

The finding that Neurogl/2 specify the subtype identity of CTNs enabled us to study the mechanisms controlling the temporal specification of this early-born population of cortical neurons. In particular we asked the question of why the Neurogenin-induced programme of CTN specification is terminated when NPCs stop generating layer 6 neurons after E12.5, although Neurog1/2 remain expressed throughout cortical neurogenesis. First, we asked whether overexpression of Neurog2 at E13.5 overrides the normal specification of E13.5 NPCs to layer 5 and 4 fates and reprogrammes these cells to a CTN fate. IUE of Neurog2 into E13.5 cortices did not induce CTN markers (Figures 4A-4D, 4G, and 4H). Instead, most Neurog2expressing cells, like control EGFP-electroporated cells, expressed the CCN marker Satb2 (Figures 4E, 4F, and 4I). This result suggests that a mechanism restricting the Neurog1/2induced CTN specification programme is already in place in E13.5 NPCs.

An alternative explanation of the above data could be that Neurog2 is inactive in E13.5 NPCs. A previous study has indeed shown that the neuronal differentiation-promoting activity of Neurog2 is significantly lower in E14.5 NPCs than E12.5 NPCs, due to phosphorylation by the GSK3 kinase that interferes with the binding of Neurog2 to its targets (Li et al., 2012). To determine whether this mechanism interferes with the CTN specification 
function of Neurogenins, we measured endogenous Neurogenin activity in E12.5 and E13.5 cortices by electroporating reporter constructs consisting of concatemerised E-boxes from the Neurod 1 and Rnd2 genes, two targets of Neurog2, driving the luciferase reporter. There was a significant reduction of the activity of the E-box reporters between E12.5 and E13.5 (Figure $4 \mathrm{~J})$, suggesting that the transcriptional activity of Neurog $1 / 2$ indeed declines immediately after the period of generation of CTNs. ChIP-PCR analysis of Neurog2 targets also showed that p300 recruitment, but not Neurog2 binding, became weaker between E12.5 and E14.5, suggesting that Neurog $1 / 2$ have a reduced capacity to recruit co-activators after the period of CTN production (Figure S4). However, when Neurog2 was co-electroporated with E-box reporters at E13.5, Neurogenin activity was increased to a level above endogenous Neurogenin activity at E12.5, which is sufficient to induce CTN fate during normal development (Figure 4J). Therefore, the inability of overexpressed Neurog2 to induce CTNs at E13.5 is not due to a reduction of its transcriptional activity. This in turn suggests that a mechanism other than the attenuation of endogenous Neurogenin activity blocks the induction of the CTN programme by Neurog1/2 at this stage.

PRC2 components are required to restrict the cortico-thalamic neuron fate to layer 6 neurons

The fact that Neurog2 overexpression at E13.5 strongly activated E-box reporters (Figure 4J) but failed to induce CTNs (Figures 4A-4I) suggested that the fate restriction of E13.5 NPCs might involve epigenetic rather than transcriptional mechanisms. Recent studies have revealed a contribution of components of Polycomb Repressive Complexes (PRCs) to the timing of generation of different cell types in the neocortex, although whether PRC proteins play a role 
in the temporal restriction of the CTN specification programme has not been addressed (Morimoto-Suzki et al., 2014; Pereira et al., 2010).

To determine whether PRCs control the timing of generation of CTNs, we first targeted Eed, an essential component of PRC2, using efficient shRNAs (Cooper and Brockdorff, 2013) (Figure S5A). While overexpressing Neurog2 alone at E13.5 did not induce CTN markers, simultaneously overexpressing Neurog2 and silencing Eed by IUE of Neurog2 and either of two different shEed constructs resulted in a marked increase in expression of CTN markers (Figures 5B, 5C, 5E, 5F, 5J, and 5K; Figures S5C-S5G). Moreover, the expression of the CCN marker Satb2 was decreased by this treatment (Figures 5H, 5I, and 5L) and many axons from Neurog2 and shEed co-electroporated neurons were found in the dorsal thalamus, while only very few axons from neurons electroporated with control vectors were found at this location (Figures 5M-5Q). Therefore, in the absence of Eed, Neurog2 could reprogramme E13.5 NPCs into proper CTNs harboring appropriate molecular markers and axonal projection patterns.

To confirm that this phenotype resulted from a loss of PRC2 activity, we also silenced Suz12 (He et al., 2012), another essential component of PRC2, while overexpressing Neurog2, and obtained similar results (Figures S5H-S5N). Collectively, these results indicate that when PRC2 function has been abolished, increased Neurog2 activity can reprogramme E13.5 NPCs into a CTN fate, a characteristic normally restricted to E12.5 NPCs.

We also examined if silencing PRC2 components alone influenced the subtype specification of E13.5 NPCs. Although knockdown of either Eed or Suz12 increased moderately the expression of Foxp2 (Figures 5A and 5J; Figures S5I and S5M), it did not alter the expression of other CTN markers including Zfpm2 (Figures 5D and 5K; Figures S5K and S5N) and Bcl11b (Figures S5B and S5E) or that of the CCN maker Satb2 (Figures 5G and 5L), 
suggesting that the loss of PRC2 function alone is not sufficient to differentiate E13.5 NPCs into CTNs.

\section{Both PRC2 and PRC1 suppress the cortico-thalamic fate in layer 4/5 NPCs}

Because of increasing evidence that PRC2 does not always act by recruiting PRC1 and promoting histone modifications (Biggar and Li, 2015), we asked whether PRC1 is also involved in the restriction of the CTN fate. Similar to the silencing of PRC2 components, silencing the PRC1 catalytic subunit RinglA (Jacob et al., 2011) (Figure S5O) and simultaneously overexpressing Neurog2 in E13.5 NPCs increased expression of CTN markers (Figures 5S and 5U-5W), while it did not have this effect when Neurog2 was not overexpressed (Figures 5R, 5T, 5V, and 5W). Knockdown of the other PRC1 catalytic subunit, RinglB (He et al., 2008) (Figure S5P) had a similar effect (Figures S5Q-S5V). These results indicate that the restriction of the CTN fate to E12.5 NPCs involves the canonical PRC2-PRC1 pathway catalysing histone modifications.

\section{The Foxp2 promoter is temporally regulated by the PRC machinery}

What is the mechanism by which PRCs suppress the CTN-inducing activity of Neurog2 in E13.5 NPCs? We first asked whether PRCs repress the Fezf2 locus, a direct and essential target of Neurog2 for CTN specification (Figure 3), which has been reported to be progressively repressed by the PRC1 component Ring1B after E14.5 (Morimoto-Suzki et al., 2014). Overexpression of Neurog2 could up-regulate Fezf2 expression as efficiently at E13.5 than at E12.5 (compare Figures 6A and 3B), suggesting that the promoter of Fezf2 is still accessible at E13.5 and that repression of Fezf2 is unlikely to account for the suppression of the CTN fate at 
this stage.

We next investigated the possibility that step(s) downstream of the induction of Fezf2 by Neurog2 in the CTN specification programme might be suppressed by PRCs at E13.5. In support of this hypothesis, overexpressing Fezf2 at E13.5 did not efficiently induce the CTN fate after 5 days (Figure 6B; Figure S6A), while inactivating PRCs by Eed knockdown while overexpressing Fezf2 markedly increased the expression of CTN markers (Figure 6C; Figure S6B), suggesting that PRCs suppress Fezf2 activity by targeting its downstream effectors.

Fezf2 is required for expression of Foxp2 in CTNs (Chen et al., 2005a; Molyneaux et al., 2005) and it binds to the promoter of the Foxp2 gene (Lodato et al., 2014), suggesting that Foxp2 is one of Fezf2 direct transcriptional targets. When Neurog2 was overexpressed and Eed silenced at E13.5, Foxp2 was up-regulated to a level higher than that seen in adjacent nonelectroporated neurons (Figures 5A-5C). Moreover, silencing of Eed or Suz12 alone was sufficient to induce Foxp2 expression (Figures 5A and 5J; Figures S5I and S5M), while other CTN markers were not affected. Together, these observations suggested that Foxp2 might be targeted by PRCs to suppress the CTN fate downstream of Neurog2 and Fezf2.

To address this possibility, we first determined whether Foxp2 is indeed involved in the specification of the CTN fate downstream of Neurog2. Silencing Foxp2 (Tsui et al., 2013) (Figures S6C and S6D) at E12.5 moderately but significantly reduced the normal generation of CTNs marked by Zfpm2 (Figures 6D, 6E, and 6G) and by axons growing into the dorsal thalamus (Figures S6E-S6I). In addition, Foxp2 knockdown efficiently blocked the induction of supernumerary CTNs by Neurog2 overexpression at E12.5 (Figures 6D, 6F, and 6G, and Figure 3R for Neurog2 + shCon). Moreover, knockdown of Foxp2 in cells overexpressing Neurog2 and silenced for Eed at E13.5 suppressed the reprogramming of electroporated 
neurons to a CTN fate (Figures $6 \mathrm{H}$ and $6 \mathrm{I}$ ) and the re-direction of axons toward the dorsal thalamus (Figures $6 \mathrm{~J}-6 \mathrm{~N}$ ). Therefore, Foxp2 is an important determinant of CTN fate downstream of Neurog2, during both normal development and reprogramming of layer $4 / 5$ neurons.

Next, we examined whether PRCs directly target Foxp2 to suppress the CTN fate after layer 6 generation. For this, we investigated PRC-dependent histone modifications at the Foxp2 promoter both during and after the period of CTN generation (E12.5 and E14.5) using chromatin immunoprecipitation assays. H3K27me3, which is deposited by PRC2, was similarly enriched at the Foxp2 promoter at E12.5 and E14.5 (Figure 6O). On the other hand, H2AK119ub, which is deposited by PRC1, was significantly more abundant at the Foxp2 promoter at E14.5 than E12.5 (Figure 6P). This suggests that PRC1 is active at the Foxp2 promoter and that this activity increases after layer 6 generation.

We next determined whether repression of Foxp2 transcription by PRCs contributes to the suppression of the CTN fate after E12.5, by asking whether forced Foxp2 expression could overcome this temporal restriction. Overexpression of Foxp2 at E13.5 was not able to induce most CTN markers, including Zfpm2 and Nfia (Figures S6J and S6K), but it could strongly induce expression of Bcl11b (Figures 6Q-6S). Moreover, Foxp2-expressing neurons extended their axons into the dorsal thalamus (Figures 6T-6V). This suggests that repression of Foxp 2 by PRCs is an important mechanism that suppresses parts of the Neurog $1 / 2$-induced CTN specification programme after layer 6 neurons have been produced. 


\section{Discussion}

In this study, we have examined the mechanisms of temporal specification of mammalian NPCs by focusing on a novel programme of cortico-thalamic neuron specification involving a Neurog1/2->Fezf2->Foxp2 regulatory cascade. We have identified two independent mechanisms that restrict this CTN specification programme to cortical NPCs generating layer 6 neurons, namely the regulation of Neurog $1 / 2$ transcriptional activity and an engagement of Polycomb repressive complexes repressing downstream genes in the CTN-specification programme. In E12.5 NPCs, Neurog1/2, which have high transcriptional activity, switch on a Fezf2-dependent genetic pathway inducing CTNs. After E12.5, the activity of Neurog1/2 is progressively reduced and simultaneously, some of the downstream genes involved in CTN specification, including Foxp2, become inaccessible because of repressive modifications catalysed by PRCs (Figure 7). Each of these mechanisms separately is sufficient to prevent E13.5 NPCs from differentiating into CTNs, revealing a high degree of robustness in the specification of cortical NPCs.

\section{Temporal regulation of NPCs in mammalian corticogenesis}

Based on extrapolation from studies in Drosophila, it has been proposed that the temporal specification of NPCs during mammalian neurogenesis involves the sequential expression of fate determinants (Kohwi and Doe, 2013). However, so far only few transcription factors have been shown to display a temporal pattern of expression during mammalian corticogenesis that is compatible with this model [i.e. Fezf2 (Hirata et al., 2004; Molyneaux et al., 2005), Otx1 (Frantz et al., 1994), Pou3fs (Dominguez et al., 2013) and Ikaros (Alsio et al., 2013)]. Moreover, the functional relevance of theses temporal expression patterns for the subtype specification of 
NPCs is yet to be determined. In particular, although transplantation experiments have argued for a restriction of the differentiation potential of NPCs during late corticogenesis (Frantz and McConnell, 1996), it is still unclear whether the commitment to a particular neuronal subtype occurs at the NPC or neuronal stage. For example, Fezf2 is expressed in NPCs at a higher level during early than late cortical development, suggesting a possible function as a temporal determinant of NPC fate. However, Fezf2 is also expressed in neurons in deep layers and the ectopic expression of Fezf 2 in upper layer neurons is able to reprogramme these neurons to a layer 5/6 subcerebral/subcortical-like fate (De la Rossa et al., 2013; Rouaux and Arlotta, 2013), suggesting that Fezf2 expression in NPCs may not be necessary for deep layer neuron specification.

Here we demonstrate that Neurog1/2, which are expressed mostly by NPCs and only transiently by postmitotic neurons (Hand et al., 2005), control the subtype specification of CTNs, suggesting that this neuronal subtype is specified at the NPC stage. A function of Neurog2 in subtype specification of deep layer cortical neurons has also been recently reported (Dennis et al., 2017). Although Neurog1/2 are expressed throughout neurogenesis, their subtype specification function operates only in a brief time window during early corticogenesis and must therefore be strictly regulated temporally. Given the lack of evidence for sequentially expressed temporal factors in NPCs, we speculate that target accessibility rather than gene expression might be temporally regulated so that NPCs are specified to different fates at different times.

\section{Foxp2 as a temporally regulated target of Polycomb Repressive Complexes}

We found in this study that PRCs temporally regulate the Foxp2 promoter, while in a previous 
work the PRC1 component RinglB has been shown to temporally regulate the promoter of Fezf2 (Morimoto-Suzki et al., 2014). Ring1B was shown to be required for the termination of subcerebral neuron production at E14.5, concomitant with an increased level of H3K27me3 modification at the Fezf2 promoter. In contrast to the suppression of the subcerebral fate at E14.5, it seemed unlikely that the suppression of the CTN fate at E13.5 also involves Fezf2 repression since this gene is active and specifies subcerebral neurons at this stage. Indeed, Neurog2 could still induce Fezf2 at E13.5, indicating that the Fezf2 promoter remains accessible. Instead, we found that the Foxp2 promoter becomes repressed by PRC1 at E13.5. These results indicate that the repression by PRCs of different targets is differentially controlled temporally, resulting in NPCs becoming restricted to different fates at different stages.

Then, how are PRCs recruited to different genomic loci at different times? We found that PRC1-dependent modifications, but not PRC2-dependent modifications, become more abundant at the Foxp2 promoter at E14.5 than E12.5, suggesting that PRC1 engagement is temporally regulated. As PRC1 complexes display considerable heterogeneity in composition (Simon and Kingston, 2013), it is possible that a specific composition of the complex enables the targeting of specific loci, in a similar way to what has been proposed for the composition of SWI/SNF or BAF complexes in NPCs versus neurons (Lessard et al., 2007). In addition, PRC1 displacement factors such as Zrf1 (Aloia et al., 2013) could be involved in this target-specific recruitment. Such regulations would make it possible that only particular genes rather than whole specification programmes, are suppressed.

\section{Temporal regulation of the transcriptional activity of Neurogenins}

In addition to the regulation of chromatin accessibility at downstream genes, we found that the 
reduction of transcriptional activity of Neurog $1 / 2$ also contributes to restricting the CTN fate after E12.5, since inactivation of PRC2 or PRC1 could re-specify E13.5 NPCs only when performed together with overexpression of Neurog2. It has been recently proposed that a reduction of Neurog2 transcriptional activity by GSK3-mediated phosphorylation accounts for the restriction of Neurog2 subtype-specification activity to deep layer neurons (Li et al., 2012). However, we found that at E13.5, Neurog1/2 retain significant transcriptional activity but have already completely lost the capacity to induce the CTN fate, indicating that a distinct mechanism operates to suppress the CTN fate. Li et al. (Li et al., 2012) also suggested that the reduction of Neurog2 activity in late corticogenesis is due to an attenuation of Neurog2 binding to its targets. However, we did not observe a significant change in Neurog2 binding between early and late corticogenesis (Figure S4A). Instead, we found reduced p300 recruitment to Neurog2-containing enhancers in late corticogenesis (Figure S4B). Post-translational regulation events [e.g. (Quan et al., 2016)] might also be involved in the progressive weakening of Neurog1/2 activity. Further investigation is required to fully characterise the mechanisms underlying the change in Neurog1/2 activity that occurs as cortical development unfolds.

\section{Additional targets of Neurog1/2}

Fezf2 has been shown to be necessary and/or sufficient for the expression of several CTNspecific genes, including Foxp2 and Zfpm2 (Chen et al., 2005a; Chen et al., 2005b; Molyneaux et al., 2005) but CTNs still retain some of their characteristics in the absence of Fezf2. The expression of Bcl11b, a transcription factor expressed broadly by subcortical projection neurons, was not altered by Fezf2 deletion (Molyneaux et al., 2005; Srinivasan et al., 2012) (see also Figure S3G), and the axons of Fezf2-deficient neurons normally reached the dorsal thalamus 
(Chen et al., 2005a; McKenna et al., 2011). CTNs are therefore less affected by loss of Fezf2 than by loss of Neurog1/2, suggesting that other Neurogenin targets might promote Fezf2independent CTN traits. Nhlhl, a potential direct target of Neurog $1 / 2$ which is expressed by intermediate progenitors during generation of layer 6 neurons, and is able to induce $B c l 11 b$ expression when overexpressed at a later stage (K.O. and F.G., unpublished observation), is such a candidate determinant of CTN fate acting downstream of Neurog $1 / 2$ and alongside Fezf2

We have shown that Foxp2 acts downstream of the Neurog $\rightarrow$ Fezf2 pathway in the CTN specification programme and that it is a target of the PRC-mediated repression terminating CTN production. Ectopic Foxp2 expression was able to induce some CTN traits, including the expression of Bcl11b and a dorsal thalamic axonal trajectory, although it was not sufficient on its own to fully reprogramme late NPCs to a CTN fate. Fezf2 directly regulates many target genes during cortical development (Lodato et al., 2014) and is likely to induce other components of the CTN specification programme, some of which might also be repressed by PRCs to terminate CTN production.

\section{Determination of cortico-thalamic or cortico-cortical fate in layer 6}

Although CTNs and CCNs are main components in layer 6 (Thomson, 2010; Tasic et al., 2016), their fate choice mechanisms have remained unclear. One possible assumption is a temporal specification mechanism of these population, which was reported recently, although the generation timing of each population is not completely separated but partially overlapping (Hatanaka et al., 2016). Our analysis, in which the fate choice during very short timing was addressed using EdU labelling and electroporation, suggested that the neurons born at around same time actually differentiate either CTNs or CCNs (if temporal specification happens strictly, 
this only labels CTNs or CCNs). Moreover, Neurog2 overexpression increased CTNs in this short time window, strongly suggesting that Neurogenin activity directly regulates the choice between CTN and CCN fate, but not the timing of differentiation. As Neurog $1 / 2$ are expressed by NPCs in an oscillatory manner (Shimojo et al., 2008), we speculate that different levels of Neurogenin activity in NPCs might contribute the genesis of CTNs and CCNs; the NPCs with high Neurogenin activity give rise to CTNs.

\section{Reprogramming cells by "unlocking" multiple mechanisms}

Reprogramming somatic cells to a given cell type (e.g. iPS cells) is inefficient and often requires multiple treatments, suggesting that the specification and maintenance of cell fates are robust processes mobilising multiple redundant mechanisms (Srivastava and DeWitt, 2016). We succeeded in reprogramming layer 4/5 NPCs into layer 6 CTN-producing NPCs by simultaneously enhancing Neurog2 activity and suppressing PRC function. Reprograming strategies can help identify essential developmental mechanisms and may contribute in the future to regenerative medicine. 


\section{Experimental Procedures}

\section{Animals and genome editing}

Mice were maintained on a 12-h light/dark cycle with free access to food and water, bred and treated according to the guidelines approved by the Home Office under the Animal (Scientific procedures) Act 1986. Protocols detailing the generation and genotyping of the genetically modified mice used have been described previously for Neurogl $1^{\text {KIGEP }}$ (Ma et al., 1998) and Neurog $2^{\text {KIGFP }}$ (Seibt et al., 2003).

To generate $F e z f 2-48 k b$ enhancer null mice, the CRISPR-Cas9 system was used as described previously (Wang et al., 2013). Short guide RNAs (sgRNAs) were identified using the MIT CRISPR Design tool to target the upstream and downstream regions of the $F e z f 2-48 \mathrm{~kb}$ enhancer, and a pair of oligonucleotides for each targeting site (Table S1) was inserted into px330, a bicistronic expression vector expressing Cas9 and sgRNA. Cas9 mRNA and the sgRNAs were produced and purified as described previously (Yang et al., 2013). Cas9 mRNA $(100 \mathrm{ng} / \mathrm{ml})$ and two sgRNAs $(25 \mathrm{ng} / \mathrm{ml})$ were injected into the cytoplasm of fertilized eggs. The deletion was confirmed by Sanger sequencing.

\section{Plasmids}

The Neurog2 expression vector was described previously (Heng et al., 2008). Fezf2 cDNA (MC202571, Origene) and Foxp2 cDNA (IMAGE ID 6851153, Source Bioscience) were subcloned into a pCAGGS plasmid. The Neurog2-binding regions were amplified by PCR from the mouse genome and subcloned into pGL4.23 to obtain firefly luciferase reporter constructs. To generate a mutation in the Fezf2-48kb E3, the E-box sequence of CAGATG was changed into TAGACT. The luciferase construct harboring tandem repeats of the E3 motif of Fezf2-48k 
and the Neurog reporters harboring Neurod1 or Rnd2 E-box were generated by inserting annealed oligonucleotides into pGL4.23 (Table S1). The shRNA constructs including shFezf2, shEed, shEed\#2, shSuz12, shRinglA, shRing1B, and shFoxp2 were also generated by inserting annealed oligonucleotides into pSuper-neo (Table S1).

\section{Immunohistochemistry}

Brains removed from embryos and pups were fixed for $1 \mathrm{~h}$ in phosphate-buffered saline (PBS) containing 4\% PFA (wt/vol), incubated overnight at $4^{\circ} \mathrm{C}$ with $20 \%$ sucrose in PBS (wt/vol), embedded in OCT compound (Sakura Finetek), and sectioned with a cryostat to obtain $14-\mu \mathrm{m}-$ thick coronal sections.

For primary antibodies, we used mouse antibody to rabbit, mouse and chick antibodies to EGFP (Molecular Probes, A11122; Santa Cruz, sc-9996; Abcam, ab13970), rat antibody to Bc111b/Ctip2 (Abcam, ab18465), mouse antibody to Satb2 (Abcam, ab51502), rabbit antibody to Tbr1 (Abcam, ab31940), rabbit antibody to Zfpm2/Fog2 (Santa Cruz, sc10755), rabbit antibody to Nfia (Aviva Systems Biology, OAAB10433), rabbit antibody to Sox5 (Abcam, ab26041), goat antibody to Foxp2 (Santa Cruz, sc-21069), rabbit antibody to Darpp32 (Chemicon, AB1656) and rabbit antibody to Nurr1 (Santa Cruz, sc-990). For detection of Bcl11b, Satb2, Zfpm2, Nfia and Sox5, antigen retrieval was performed by incubating the sections for $20 \mathrm{~min}$ at $90^{\circ} \mathrm{C}$ in $0.01 \mathrm{M}$ sodium citrate buffer $(\mathrm{pH}$ 6.0). Because EGFP fluorescence disappeared by antigen retrieval treatment, EGFP was immunostained with chick or moue antibody against EGFP for re-visualization. Immune complexes were detected with Alexa Flour-conjugated secondary antibodies (Molecular Probes). For nuclear staining, 2 $\mu \mathrm{g} / \mathrm{ml}$ DAPI (Molecular Probes) was used. Images were acquired using a confocal microscope 
(SP5, Leica).

\section{In utero electroporation and EdU administration}

In utero electroporation of the cerebral cortex was performed in anesthetized time-pregnant mice, as previously described (Pacary et al., 2011). Cortices were electroporated with five 50ms electrical pulses at $31 \mathrm{~V}$ with 1 -s intervals using 3-mm platinum electrodes (Harvard Apparatus). For injection of EdU (5-ethynyl-2'-deoxyuridine), the pregnant mice were injected intraperitoneally with a EdU solution (Santa Cruz, $20 \mathrm{mg}$ per $\mathrm{kg}$ body weight). EdU was detected using ClickiT ${ }^{\circledR}$ EdU Imaging kit (Invitrogen).

\section{RNA isolation and qRT-PCR}

Total RNA was extracted using the Nucleobond RNA extraction kit (Macherey Nagel), and subjected to reverse transcription using the High-Capacity RNA-to-cDNA ${ }^{\mathrm{TM}}$ kit (Applied Biosystems). Gene expression was detected using TaqMan Gene expression assays (Applied Biosystems) and a 7500 real-time PCR system (Applied Biosystems). Data was analyzed using standard protocols to calculate relative expression with the ddCT method using Ppia as an endogenous control. Each probe was analyzed in duplicates for at least 3 independent samples per group.

\section{Cell culture and transfection}

Mouse teratocarcinoma P19 cells were cultured in high-glucose DMEM (Invitrogen) supplemented with $10 \%$ fetal bovine serum, $2 \mathrm{mM}$ glutamine and $1 \%$ penicillin/streptomycin. Mouse neural stem cell line NS-5 cells were cultured in Euromed-N medium (Euroclone) 
supplemented with 1\% N2 (Invitrogen), epidermal growth factor, basic fibroblast growth factor (Peprotech, both at $10 \mathrm{ng} / \mathrm{ml}$ ), and $2 \mu \mathrm{g} / \mathrm{ml}$ laminin (Sigma). Cells were plated in 24-well or 48-well plates and transfected with Lipofectamine 2000 reagent according to the manufacturer's protocol (Invitrogen). Twenty-four hours after transfection, cells were collected for subsequent analyses.

\section{Luciferase assay}

NS-5 cells and cortical cells were transfected with various firefly luciferase constructs together with pGL4.74 (a thymidine kinase promoter-driven Renilla luciferase reporter).

The luciferase activities of the cell lysates were measured using the Dual-Luciferase reporter assay system (Promega). The firefly luciferase activity was normalized by the Renilla luciferase activity. For in vivo luciferase assay, cortical cells were electroporated with various luciferase vectors, the control Renilla luciferase vector and EGFP expression vector. EGFP-positive areas of the electroporated brains were dissected out and lysed.

\section{Chromatin immunoprecipitation (ChIP) assay}

The dorsolateral portions of the neocortex were dissected out, fixed sequentially with di(Nsuccinimidyl)-glutarate and 1\% formaldehyde in phosphate-buffered saline, lysed in SDS lysis buffer $(0.5 \%$ SDS, $10 \mathrm{mM}$ EDTA, $50 \mathrm{mM}$ Tris- $\mathrm{HCl} \mathrm{pH} 8.0)$ supplemented with protease inhibitor cocktail (Roche), sonicated for $20 \mathrm{~min}$. Immunoprecipitations were performed as described previously (Martynoga et al., 2013). Quantities of immunoprecipitated DNA were calculated by comparison with a standard curve generated by serial dilutions of input DNA using a 7500 real-time PCR system (Applied Biosystems) and a SYBR green-based kit for 
quantitative PCR (iQ Supermix, Bio-Rad). Antibodies and amounts used for $20 \mu \mathrm{g}$ chromatin: rabbit anti-H3K27me3 (0.25 $\mu \mathrm{g}$, Millipore, 07-449), rabbit anti-H2AK119ub (0.25 $\mu \mathrm{g}$, Cell Signaling, D27C4), goat anti-Neurog2 (0.5 $\mu \mathrm{g}$, Santa Cruz, sc-19233) or rabbit anti-p300 (0.5 $\mu g$, Santa Cruz, sc-585). The primers used are summarized in Table S1.

\section{Quantification of axon projection}

The EGFP fluorescence signals in the ventrobasal nucleus of the dorsal thalamus of three or four sections were measured, and the average value was considered as the axon amount in the dorsal thalamus. The values were then normalised by the similarly averaged EGFP fluorescence signals in the somatosensory area of the cortex to adjust the electroporation efficiency among different brains.

\section{Statistical analysis}

Unless indicated otherwise, data were represented as means \pm SEM or SD of values from at least three embryos from at least two litters. For quantification of in vivo cell counting, all positive cells were counted in the areas where rostro-caudal and medio-lateral levels were carefully matched between animals. For two-group comparisons with equal variance as determined by the $F$-test, an unpaired Student's $t$ test was used. Welch's correction was used for unpaired t-tests of normally distributed data with unequal variance. Differences between groups were considered to be significant at $p<0.05$. Each $p$ value was stated in figures or figure legends. 


\section{Acknowledgments}

We gratefully acknowledge Sophie Wood and Graham Preece for technical support, Rekha Subramaniams and Jacek Mor for managing the mouse colony, and members of the Guillemot lab for discussions. The study was conceived and the manuscript was written by K.O. and F.G. K.O. performed most of the work while D.L.C.B. helped with ChIP experiments. K.O. was supported by fellowships from the Uehara memorial foundation, the UK Medical Research Council (MRC) and the Francis Crick Institute and D.L.C.B. by fellowships from the Federation of European Biochemical Societies and the Francis Crick Institute. This work was supported by the Francis Crick Institute, which receives its core funding from Cancer Research UK (FC0010089), the UK Medical Research Council (FC0010089), and the Wellcome Trust (FC0010089); by the UK Biotechnology and Biological Sciences Research Council (project grant BB/K005316/1 to F.G.); by the UK Medical Research Council (project grant U117570528 to F.G.); by the MEXT/JSPS (KAKENHI JP17K07061 to K.O.); by the Takeda Science Foundation (project grant to K.O.), and by the Wellcome Trust (investigator award 106187/Z/14/Z to F.G.). The authors declare no conflict of interest. 


\section{References}

Aloia, L., Di Stefano, B., and Di Croce, L. (2013). Polycomb complexes in stem cells and embryonic development. Development 140, 2525-2534.

Alsio, J.M., Tarchini, B., Cayouette, M., and Livesey, F.J. (2013). Ikaros promotes early-born neuronal fates in the cerebral cortex. Proc Natl Acad Sci U S A 110, E716-725.

Arimatsu, Y., Ishida, M., Kaneko, T., Ichinose, S., and Omori, A. (2003). Organization and development of corticocortical associative neurons expressing the orphan nuclear receptor Nurr1. J Comp Neurol 466, 180-196.

Arlotta, P., Molyneaux, B.J., Chen, J., Inoue, J., Kominami, R., and Macklis, J.D. (2005).

Neuronal subtype-specific genes that control corticospinal motor neuron development in vivo. Neuron 45, 207-221.

Betancourt, J., Katzman, S., and Chen, B. (2014). Nuclear factor one B regulates neural stem cell differentiation and axonal projection of corticofugal neurons. J Comp Neurol 522, 6-35. Biggar, K.K., and Li, S.S. (2015). Non-histone protein methylation as a regulator of cellular signalling and function. Nat Rev Mol Cell Biol 16, 5-17.

Blackledge, N.P., Rose, N.R., and Klose, R.J. (2015). Targeting Polycomb systems to regulate gene expression: modifications to a complex story. Nat Rev Mol Cell Biol 16, 643-649.

Castro, D.S., Skowronska-Krawczyk, D., Armant, O., Donaldson, I.J., Parras, C., Hunt, C., Critchley, J.A., Nguyen, L., Gossler, A., Gottgens, B., et al. (2006). Proneural bHLH and Brn proteins coregulate a neurogenic program through cooperative binding to a conserved DNA motif. Dev Cell 11, 831-844.

Chen, B., Schaevitz, L.R., and McConnell, S.K. (2005a). Fezl regulates the differentiation and axon targeting of layer 5 subcortical projection neurons in cerebral cortex. Proc Natl Acad Sci U S A 102, 17184-17189.

Chen, J.G., Rasin, M.R., Kwan, K.Y., and Sestan, N. (2005b). Zfp312 is required for subcortical axonal projections and dendritic morphology of deep-layer pyramidal neurons of the cerebral cortex. Proc Natl Acad Sci U S A 102, 17792-17797.

Cong, L., Ran, F.A., Cox, D., Lin, S., Barretto, R., Habib, N., Hsu, P.D., Wu, X., Jiang, W., Marraffini, L.A., et al. (2013). Multiplex genome engineering using CRISPR/Cas systems. Science 339, 819-823.

Cooper, S., and Brockdorff, N. (2013). Genome-wide shRNA screening to identify factors mediating Gata6 repression in mouse embryonic stem cells. Development 140, 4110-4115. De la Rossa, A., Bellone, C., Golding, B., Vitali, I., Moss, J., Toni, N., Luscher, C., and Jabaudon, D. (2013). In vivo reprogramming of circuit connectivity in postmitotic neocortical 
neurons. Nat Neurosci 16, 193-200.

Dennis, D.J., Wilkinson, G., Li, S., Dixit, R., Adnani, L., Balakrishnan, A., Han, S., Kovach, C., Gruenig, N., Kurrasch, D.M., et al. (2017). Neurog2 and Ascl1 together regulate a postmitotic derepression circuit to govern laminar fate specification in the murine neocortex. Proc Natl Acad Sci U S A 114, E4934-E4943.

Dominguez, M.H., Ayoub, A.E., and Rakic, P. (2013). POU-III transcription factors (Brn1, Brn2, and Oct6) influence neurogenesis, molecular identity, and migratory destination of upper-layer cells of the cerebral cortex. Cereb Cortex 23, 2632-2643.

Elnitski, L., Hardison, R.C., Li, J., Yang, S., Kolbe, D., Eswara, P., O'Connor, M.J., Schwartz, S., Miller, W., and Chiaromonte, F. (2003). Distinguishing regulatory DNA from neutral sites. Genome Res 13, 64-72.

Florio, M., and Huttner, W.B. (2014). Neural progenitors, neurogenesis and the evolution of the neocortex. Development 141, 2182-2194.

Frantz, G.D., and McConnell, S.K. (1996). Restriction of late cerebral cortical progenitors to an upper-layer fate. Neuron 17, 55-61.

Frantz, G.D., Weimann, J.M., Levin, M.E., and McConnell, S.K. (1994). Otx1 and Otx2 define layers and regions in developing cerebral cortex and cerebellum. J Neurosci 14, 5725 5740 .

Galazo, M.J., Emsley, J.G., and Macklis, J.D. (2016). Corticothalamic Projection Neuron Development beyond Subtype Specification: Fog2 and Intersectional Controls Regulate Intraclass Neuronal Diversity. Neuron 91, 90-106.

Gohlke, J.M., Armant, O., Parham, F.M., Smith, M.V., Zimmer, C., Castro, D.S., Nguyen, L., Parker, J.S., Gradwohl, G., Portier, C.J., et al. (2008). Characterization of the proneural gene regulatory network during mouse telencephalon development. BMC Biol 6, 15 .

Greig, L.C., Woodworth, M.B., Galazo, M.J., Padmanabhan, H., and Macklis, J.D. (2013). Molecular logic of neocortical projection neuron specification, development and diversity. Nat Rev Neurosci 14, 755-769.

Grossniklaus, U., and Paro, R. (2014). Transcriptional silencing by polycomb-group proteins. Cold Spring Harb Perspect Biol 6, a019331.

Han, W., Kwan, K.Y., Shim, S., Lam, M.M., Shin, Y., Xu, X., Zhu, Y., Li, M., and Sestan, N. (2011). TBR1 directly represses Fezf2 to control the laminar origin and development of the corticospinal tract. Proc Natl Acad Sci U S A 108, 3041-3046.

Hand, R., Bortone, D., Mattar, P., Nguyen, L., Heng, J.I., Guerrier, S., Boutt, E., Peters, E., Barnes, A.P., Parras, C., et al. (2005). Phosphorylation of Neurogenin2 specifies the migration properties and the dendritic morphology of pyramidal neurons in the neocortex. Neuron 48 , 
$45-62$.

Hatanaka, Y., Namikawa, T., Yamauchi, K., and Kawaguchi, Y. (2016). Cortical Divergent Projections in Mice Originate from Two Sequentially Generated, Distinct Populations of Excitatory Cortical Neurons with Different Initial Axonal Outgrowth Characteristics. Cereb Cortex 26, 2257-2270.

He, A., Ma, Q., Cao, J., von Gise, A., Zhou, P., Xie, H., Zhang, B., Hsing, M., Christodoulou, D.C., Cahan, P., et al. (2012). Polycomb repressive complex 2 regulates normal development of the mouse heart. Circ Res 110, 406-415.

He, J., Kallin, E.M., Tsukada, Y., and Zhang, Y. (2008). The H3K36 demethylase $\mathrm{Jhdm} 1 \mathrm{~b} / \mathrm{Kdm} 2 \mathrm{~b}$ regulates cell proliferation and senescence through $\mathrm{p} 15$ (Ink4b). Nat Struct Mol Biol 15, 1169-1175.

Heng, J.I., Nguyen, L., Castro, D.S., Zimmer, C., Wildner, H., Armant, O., SkowronskaKrawczyk, D., Bedogni, F., Matter, J.M., Hevner, R., et al. (2008). Neurogenin 2 controls cortical neuron migration through regulation of Rnd2. Nature 455, 114-118.

Hirata, T., Suda, Y., Nakao, K., Narimatsu, M., Hirano, T., and Hibi, M. (2004). Zinc finger gene fez-like functions in the formation of subplate neurons and thalamocortical axons. Dev Dyn 230, 546-556.

Hisaoka, T., Nakamura, Y., Senba, E., and Morikawa, Y. (2010). The forkhead transcription factors, Foxp1 and Foxp2, identify different subpopulations of projection neurons in the mouse cerebral cortex. Neuroscience 166, 551-563.

Huang, H.P., Liu, M., El-Hodiri, H.M., Chu, K., Jamrich, M., and Tsai, M.J. (2000). Regulation of the pancreatic islet-specific gene BETA2 (neuroD) by neurogenin 3. Mol Cell Biol 20, 3292-3307.

Jacob, E., Hod-Dvorai, R., Ben-Mordechai, O.L., Boyko, Y., and Avni, O. (2011). Dual function of polycomb group proteins in differentiated murine T helper (CD4+) cells. J Mol Signal 6, 5.

Joshi, P.S., Molyneaux, B.J., Feng, L., Xie, X., Macklis, J.D., and Gan, L. (2008). Bhlhb5 regulates the postmitotic acquisition of area identities in layers II-V of the developing neocortex. Neuron 60, 258-272.

Kohwi, M., and Doe, C.Q. (2013). Temporal fate specification and neural progenitor competence during development. Nat Rev Neurosci 14, 823-838.

Kwan, K.Y., Lam, M.M., Krsnik, Z., Kawasawa, Y.I., Lefebvre, V., and Sestan, N. (2008). SOX 5 postmitotically regulates migration, postmigratory differentiation, and projections of subplate and deep-layer neocortical neurons. Proc Natl Acad Sci U S A 105, 16021-16026. Lessard, J., Wu, J.I., Ranish, J.A., Wan, M., Winslow, M.M., Staahl, B.T., Wu, H., Aebersold, 
R., Graef, I.A., and Crabtree, G.R. (2007). An essential switch in subunit composition of a chromatin remodeling complex during neural development. Neuron 55, 201-215.

Li, S., Mattar, P., Zinyk, D., Singh, K., Chaturvedi, C.P., Kovach, C., Dixit, R., Kurrasch, D.M., Ma, Y.C., Chan, J.A., et al. (2012). GSK3 temporally regulates neurogenin 2 proneural activity in the neocortex. J Neurosci 32, 7791-7805.

Lodato, S., and Arlotta, P. (2015). Generating neuronal diversity in the mammalian cerebral cortex. Annu Rev Cell Dev Biol 31, 699-720.

Lodato, S., Molyneaux, B.J., Zuccaro, E., Goff, L.A., Chen, H.H., Yuan, W., Meleski, A., Takahashi, E., Mahony, S., Rinn, J.L., et al. (2014). Gene co-regulation by Fezf2 selects neurotransmitter identity and connectivity of corticospinal neurons. Nat Neurosci 17, 10461054.

Loots, G.G., Locksley, R.M., Blankespoor, C.M., Wang, Z.E., Miller, W., Rubin, E.M., and Frazer, K.A. (2000). Identification of a coordinate regulator of interleukins 4, 13, and 5 by cross-species sequence comparisons. Science 288, 136-140.

Ma, Q., Chen, Z., del Barco Barrantes, I., de la Pompa, J.L., and Anderson, D.J. (1998). neurogenin 1 is essential for the determination of neuronal precursors for proximal cranial sensory ganglia. Neuron 20, 469-482.

Magdaleno, S., Jensen, P., Brumwell, C.L., Seal, A., Lehman, K., Asbury, A., Cheung, T., Cornelius, T., Batten, D.M., Eden, C., et al. (2006). BGEM: an in situ hybridization database of gene expression in the embryonic and adult mouse nervous system. PLoS Biol 4, e86. Mali, P., Yang, L., Esvelt, K.M., Aach, J., Guell, M., DiCarlo, J.E., Norville, J.E., and Church, G.M. (2013). RNA-guided human genome engineering via Cas9. Science 339, 823-826.

Margueron, R., and Reinberg, D. (2011). The Polycomb complex PRC2 and its mark in life. Nature 469, 343-349.

Martynoga, B., Drechsel, D., and Guillemot, F. (2012). Molecular control of neurogenesis: a view from the mammalian cerebral cortex. Cold Spring Harb Perspect Biol 4.

Martynoga, B., Mateo, J.L., Zhou, B., Andersen, J., Achimastou, A., Urban, N., van den Berg, D., Georgopoulou, D., Hadjur, S., Wittbrodt, J., et al. (2013). Epigenomic enhancer annotation reveals a key role for NFIX in neural stem cell quiescence. Genes Dev 27, 17691786.

McConnell, S.K. (1995). Constructing the cerebral cortex: neurogenesis and fate determination. Neuron 15, 761-768.

McConnell, S.K., and Kaznowski, C.E. (1991). Cell cycle dependence of laminar determination in developing neocortex. Science 254, 282-285.

McKenna, W.L., Betancourt, J., Larkin, K.A., Abrams, B., Guo, C., Rubenstein, J.L., and 
Chen, B. (2011). Tbr1 and Fezf2 regulate alternate corticofugal neuronal identities during neocortical development. J Neurosci 31, 549-564.

Molyneaux, B.J., Arlotta, P., Hirata, T., Hibi, M., and Macklis, J.D. (2005). Fezl is required for the birth and specification of corticospinal motor neurons. Neuron 47, 817-831.

Molyneaux, B.J., Arlotta, P., Menezes, J.R., and Macklis, J.D. (2007). Neuronal subtype specification in the cerebral cortex. Nat Rev Neurosci 8, 427-437.

Morimoto-Suzki, N., Hirabayashi, Y., Tyssowski, K., Shinga, J., Vidal, M., Koseki, H., and Gotoh, Y. (2014). The polycomb component Ring1B regulates the timed termination of subcerebral projection neuron production during mouse neocortical development.

Development 141, 4343-4353.

Nieto, M., Schuurmans, C., Britz, O., and Guillemot, F. (2001). Neural bHLH genes control the neuronal versus glial fate decision in cortical progenitors. Neuron 29, 401-413.

Oishi, K., Aramaki, M., and Nakajima, K. (2016a). Mutually repressive interaction between Brn1/2 and Rorb contributes to the establishment of neocortical layer 2/3 and layer 4. Proc Natl Acad Sci U S A 113, 3371-3376.

Oishi, K., Nakagawa, N., Tachikawa, K., Sasaki, S., Aramaki, M., Hirano, S., Yamamoto, N., Yoshimura, Y., and Nakajima, K. (2016b). Identity of neocortical layer 4 neurons is specified through correct positioning into the cortex. Elife 5.

Ovcharenko, I., Nobrega, M.A., Loots, G.G., and Stubbs, L. (2004). ECR Browser: a tool for visualizing and accessing data from comparisons of multiple vertebrate genomes. Nucleic Acids Res 32, W280-286.

Pacary, E., Heng, J., Azzarelli, R., Riou, P., Castro, D., Lebel-Potter, M., Parras, C., Bell, D.M., Ridley, A.J., Parsons, M., et al. (2011). Proneural transcription factors regulate different steps of cortical neuron migration through Rnd-mediated inhibition of RhoA signaling. Neuron 69, 1069-1084.

Pereira, J.D., Sansom, S.N., Smith, J., Dobenecker, M.W., Tarakhovsky, A., and Livesey, F.J. (2010). Ezh2, the histone methyltransferase of PRC2, regulates the balance between selfrenewal and differentiation in the cerebral cortex. Proc Natl Acad Sci U S A 107, 1595715962.

Quan, X.J., Yuan, L., Tiberi, L., Claeys, A., De Geest, N., Yan, J., van der Kant, R., Xie, W.R., Klisch, T.J., Shymkowitz, J., et al. (2016). Post-translational Control of the Temporal Dynamics of Transcription Factor Activity Regulates Neurogenesis. Cell 164, 460-475. Rouaux, C., and Arlotta, P. (2013). Direct lineage reprogramming of post-mitotic callosal neurons into corticofugal neurons in vivo. Nat Cell Biol 15, 214-221.

Schuurmans, C., Armant, O., Nieto, M., Stenman, J.M., Britz, O., Klenin, N., Brown, C., 
Langevin, L.M., Seibt, J., Tang, H., et al. (2004). Sequential phases of cortical specification involve Neurogenin-dependent and -independent pathways. EMBO J 23, 2892-2902.

Seibt, J., Schuurmans, C., Gradwhol, G., Dehay, C., Vanderhaeghen, P., Guillemot, F., and Polleux, F. (2003). Neurogenin 2 specifies the connectivity of thalamic neurons by controlling axon responsiveness to intermediate target cues. Neuron 39, 439-452.

Seo, S., Lim, J.W., Yellajoshyula, D., Chang, L.W., and Kroll, K.L. (2007). Neurogenin and NeuroD direct transcriptional targets and their regulatory enhancers. EMBO J 26, 5093-5108. Sessa, A., Ciabatti, E., Drechsel, D., Massimino, L., Colasante, G., Giannelli, S., Satoh, T., Akira, S., Guillemot, F., and Vania, B. (2016). The Tbr2 Molecular Network Controls Cortical Neuronal Differentiation Through Complementary Genetic and Epigenetic Pathways. Cereb Cortex.

Shim, S., Kwan, K.Y., Li, M., Lefebvre, V., and Sestan, N. (2012). Cis-regulatory control of corticospinal system development and evolution. Nature 486, 74-79.

Shimojo, H., Ohtsuka, T., and Kageyama, R. (2008). Oscillations in notch signaling regulate maintenance of neural progenitors. Neuron 58, 52-64.

Simon, J.A., and Kingston, R.E. (2013). Occupying chromatin: Polycomb mechanisms for getting to genomic targets, stopping transcriptional traffic, and staying put. Mol Cell 49, 808824.

Srinivasan, K., Leone, D.P., Bateson, R.K., Dobreva, G., Kohwi, Y., Kohwi-Shigematsu, T., Grosschedl, R., and McConnell, S.K. (2012). A network of genetic repression and derepression specifies projection fates in the developing neocortex. Proc Natl Acad Sci U S A 109, 19071-19078.

Srivastava, D., and DeWitt, N. (2016). In Vivo Cellular Reprogramming: The Next Generation. Cell 166, 1386-1396.

Sun, T., and Hevner, R.F. (2014). Growth and folding of the mammalian cerebral cortex: from molecules to malformations. Nat Rev Neurosci 15, 217-232.

Tasic, B., Menon, V., Nguyen, T.N., Kim, T.K., Jarsky, T., Yao, Z., Levi, B., Gray, L.T., Sorensen, S.A., Dolbeare, T., et al. (2016). Adult mouse cortical cell taxonomy revealed by single cell transcriptomics. Nat Neurosci 19, 335-346.

Thomson, A.M. (2010). Neocortical layer 6, a review. Front Neuroanat 4, 13. Tsui, D., Vessey, J.P., Tomita, H., Kaplan, D.R., and Miller, F.D. (2013). FoxP2 regulates neurogenesis during embryonic cortical development. J Neurosci 33, 244-258.

Wang, H., Yang, H., Shivalila, C.S., Dawlaty, M.M., Cheng, A.W., Zhang, F., and Jaenisch, R. (2013). One-step generation of mice carrying mutations in multiple genes by CRISPR/Casmediated genome engineering. Cell 153,910-918. 
Watakabe, A., Ichinohe, N., Ohsawa, S., Hashikawa, T., Komatsu, Y., Rockland, K.S., and Yamamori, T. (2007). Comparative analysis of layer-specific genes in Mammalian neocortex. Cereb Cortex 17, 1918-1933.

Yang, H., Wang, H., Shivalila, C.S., Cheng, A.W., Shi, L., and Jaenisch, R. (2013). One-step generation of mice carrying reporter and conditional alleles by CRISPR/Cas-mediated genome engineering. Cell 154, 1370-1379.

Yoon, K.J., Vissers, C., Ming, G.L., and Song, H. (2018). Epigenetics and epitranscriptomics in temporal patterning of cortical neural progenitor competence. J Cell Biol 217, 1901-1914. Zahr, S.K., Yang, G., Kazan, H., Borrett, M.J., Yuzwa, S.A., Voronova, A., Kaplan, D.R., and Miller, F.D. (2018). A Translational Repression Complex in Developing Mammalian Neural Stem Cells that Regulates Neuronal Specification. Neuron 97, 520-537 e526. 


\section{Figure Legends}

\section{Figure 1. Inactivation of Neurog1/2 decreases generation of cortico-thalamic neurons}

(A-L) Immunostaining for the indicated subtype-specific makers in E18.5 cortices of control, Neurog2KO, and Neurog1/2KO mice. Tbr1 is generally used as a marker for CTNs but we observed that Tbr1 is also expressed by CCNs at moderate levels (data not shown). (M and N) The numbers of Bcl11b (M)- or Satb2 (N)-positive cells in 220- $\mu$ m width cortical columns of the indicated genotypes was counted (Control, $\mathrm{n}=4 ;$ Neurog2KO, $\mathrm{n}=4 ;$ Neurogl $2 \mathrm{KO}, \mathrm{n}=3$ ). (O) The number of cells visualized with DAPI in layer 6 of the indicated genotypes was counted (Control, $\mathrm{n}=4 ;$ Neurog2KO, $\mathrm{n}=4 ; \operatorname{Neurog} 1 / 2 K O, \mathrm{n}=3$ ). (P) Quantification of the percentage of Bcl11b-postive cells in layer 6 (Control, $\mathrm{n}=4 ; \operatorname{Neurog} 2 K O, \mathrm{n}=4 ; \operatorname{Neurog} 1 / 2 K O, \mathrm{n}=3$ ). (Q and R) The number of Foxp2 (Q)- or Zfpm2 (R)-positive cells in the indicated genotypes was counted (Control, $\mathrm{n}=4 ;$ Neurog2KO, $\mathrm{n}=4 ;$ Neurog $1 / 2 K O, \mathrm{n}=4)$. Scale bar: $50 \mu \mathrm{m}$. Data are represented as means $\pm \mathrm{SD} ;{ }^{*} p<0.005,{ }^{* *} p<0.001, * * * p<0.0001$ from two-tailed unpaired Student's $t$-test with Welch's correction.

\section{Figure 2. Neurog2 overexpression promotes cortico-thalamic neuron specification}

(A-F) Immunostaining for the indicated subtype-specific markers in E18.5 cortices after in utero electroporation at E12.5 of EGFP and control (A, C, and E) or Neurog2 expression vector (B, D, and F). (G-I) Quantification of the percentage of marker-positive cells among EGFPpositive cells in (A)-(F) (Control, $n=4 ;$ Neurog2, $n=4)$. (J-M) Immunostaining for EGFP in P1 brains after in utero electroporation as in (A) and (B). A representative image is shown in each condition. An anterior level of the electroporated brains is presented to demonstrate electroporation efficiency ( $\mathrm{J}$ and L). A posterior level including the dorsal thalamus (indicated 
with dotted circles) is presented to show the axon trajectories of the electroporated cells ( $\mathrm{K}$ and M). Quantification of $(\mathrm{J}-\mathrm{M})$ is shown $(\mathrm{N})$ (Control, $\mathrm{n}=4$; Neurog2, $\mathrm{n}=3)$. (O-R) EdU was administrated 1 hour before in utero electroporation at E12.5, and the brains were analyzed at E18.5 (O). EdU staining together with EGFP immunostaining (left panels) and Zfpm2 immunostaining (right panels) are shown (P and Q). The percentage of Zfpm2-positive cells among both EdU- and EGFP-positive cells shown in (P) and (Q) was calculated (R) (Control, $\mathrm{n}=4$; Neurog2, $\mathrm{n}=3)$. Scale bars: $50 \mu \mathrm{m}(\mathrm{A}$ and $\mathrm{P}) ; 500 \mu \mathrm{m}(\mathrm{J})$. Data are represented as means $\pm \mathrm{SEM} ;{ }^{*} p<0.005 ;{ }^{* *} p<0.001 ;{ }^{* *} p<0.0001$ from two-tailed unpaired Student's $t$-test with Welch's correction.

\section{Figure 3. Fezf2 is a target of Neurog2 for cortico-thalamic neuron specification}

(A) A list of candidate Neurog1/2 target genes, that have decreased expression levels in Neurog2 and Neurog1/2 mutant cortices and Neurog2 ChIP-Seq peaks in their vicinity. The numbers of conserved ChIP-Seq peaks in each gene between mouse and human are also shown. (B) The mRNA levels of the indicated genes, normalized by that of Ppia, are shown. Samples were collected by FACS from E16.5 cortices that had been electroporated at E12.5 with EGFP or Neurog2 expression vectors (Control, $\mathrm{n}=3$; Neurog2, $\mathrm{n}=3$ ). (C) Trancriptional activation of Neurog2-binding genomic elements by Neurog2 and Ascll. Neurog2-binding elements were cloned into a luciferase vector harboring a minimal promoter and transfected in NS5 cells together with control, Neurog2 or Ascll expression vector, and their luciferase activities were measured. Luciferase activity was normalized by the Renilla luciferase activity (Control, $\mathrm{n}=3$; Neurog2, $\mathrm{n}=3$; Ascl1 $\mathrm{n}=3$ ). (D) The DNA sequence of the Neurog2-binding region $48 \mathrm{~kb}$ upstream of the transcription start site of the Fezf2 gene contains five E-box motif(E1-E5). (E) 
The transcriptional activity of tandem repeats of the E3 motif was measured as in (C) (Control, $\mathrm{n}=3$; Neurog2, $\mathrm{n}=3 ;$ Ascl1 $\mathrm{n}=3$ ). (F) The activity of the Fezf2-48kb element with mutations in the E3 motif $[F e z f 2-48 k b(m u t)]$ was measured as in $(\mathrm{C})(\mathrm{n}=3$ in each condition). (G) E12.5 cortices were electroporated with the indicated luciferase vectors. After 1 day, the brains were lysed and subjected to luciferase analysis (miniP, $\mathrm{n}=3 ; F e z f 2-48 k b, \mathrm{n}=4 ; F e z f 2-48 k b(m u t), \mathrm{n}$ =4). (H) E12.5 cortices were electroporated with the Fezf2-48kb luciferase vector together with a control shRNA (shCon) or Neurog2 shRNA (shNeurog2) (shCon, $\mathrm{n}=4 ;$ shNeurog2, $\mathrm{n}=4)$. (I) Expression level of Fezf2 mRNA in E14.5 cortices of control or Fezf2-48kb element knockout mice (heterozygous control, $\mathrm{n}=3$; homozygous mutant, $\mathrm{n}=5$ ). $(\mathrm{J}-\mathrm{O})$ Immunostaining for the indicated subtype-specific markers in E18.5 cortices after in utero electroporation at E12.5 of EGFP and Fezf2 expression vector (J, L, and N). Quantification of the percentage of marker-positive cells among EGFP-positive cells (K, M, and O) (for Foxp2 and Satb2, Control, $\mathrm{n}=4 ;$ Fezf2, $\mathrm{n}=3 \mid$ for Zfpm2, Control, $\mathrm{n}=4 ;$ Fezf2, $\mathrm{n}=4)$. (P-U) Experiments were performed as in $(\mathrm{J})-(\mathrm{M})$ with transfection of the indicated plasmids (shCon + Control, $\mathrm{n}=4 ; \operatorname{shFezf2}+$ Control, $\mathrm{n}=4 ;$ shCon + Neurog $2, \mathrm{n}=3 ; \operatorname{shFezf} 2+$ Neurog $2, \mathrm{n}=4)$. Scale bars: $50 \mu \mathrm{m}(\mathrm{J}$ and $\mathrm{P})$. Data are represented as means $\pm \mathrm{SEM} ;{ }^{*} p<0.05 ; * * p<0.005$; ${ }^{* * *} p<0.0005$ from two-tailed unpaired Student's $t$-test with Welch's correction; ns, not significant.

\section{Figure 4. Neurog2 overexpression in E13.5 NPCs fails to induce cortico-thalamic neurons}

(A-F) Immunostaining for the indicated subtype-specific markers in E18.5 cortices after in utero electroporation at E13.5 of EGFP and control (A, C, and E) or Neurog2 expression vector (B, D, and F). (G-I) Quantification of the percentage of marker-positive cells among EGFP- 
positive cells in (A)-(F) (Control, $n=4 ;$ Neurog2, $n=4)$. (J) E12.5 or E13.5 cortices were electroporated with Neurog reporters containing tandem repeats of E-box motifs (Neurod1Ebox, Rnd2Ebox). E13.5 cortices were also co-electroporated with Neurog2 expression vector together with the Neurog reporters. After 1 day, the brains were lysed and subjected to luciferase analysis (for Neurod1Ebox: E12.5, $\mathrm{n}=5$; E13.5, $\mathrm{n}=6$; E13.5 with Neurog2, $\mathrm{n}=4$; for Rnd2Ebox: E12.5, $\mathrm{n}=4$; E13.5, $\mathrm{n}=4$; E13.5 with Neurog2, $\mathrm{n}=4$ ). Scale bar: $50 \mu \mathrm{m}$. Data are represented as means $\pm \mathrm{SEM} ;{ }^{*} p<0.05 ; * * p<0.005 ; * * * p<0.0001$ from two-tailed unpaired Student's $t$-test with Welch's correction; ns, not significant.

Figure 5. Neurog2 reprogrammes E13.5 NPCs to a cortico-thalamic fate when PRC2 or

\section{PRC1 is inactivated}

(A-I) Immunostaining for the indicated subtype-specific markers in E18.5 cortices after in utero electroporation at E13.5 of EGFP and the indicated plasmids. (J-L) Quantification of the percentage of marker-positive cells among EGFP-positive cells in (A)-(I) (for Foxp2, shCon + Control, $\mathrm{n}=4 ;$ shEed + Control, $\mathrm{n}=4 ; \operatorname{shCon}+$ Neurog $2, \mathrm{n}=4 ; \operatorname{shEed}+$ Neurog $2, \mathrm{n}=4 \mid$ for Zfpm2, shCon + Control, $\mathrm{n}=4 ;$ shEed + Control, $\mathrm{n}=4 ;$ shCon + Neurog $2, \mathrm{n}=4 ;$ shEed + Neurog2, $\mathrm{n}=3 \mid$ for Satb2, shCon + Control, $\mathrm{n}=4 ;$ shEed + Control, $\mathrm{n}=4 ;$ shCon + Neurog 2 , $\mathrm{n}=3$; shEed + Neurog2, $\mathrm{n}=4)$. (M-Q) Immunostaining for EGFP in P3 brains after in utero electroporation at E13.5 of EGFP and the indicated plasmids. A representative image is shown in each condition. Dotted circles at a posterior level indicate the dorsal thalamus ( $\mathrm{N}$ and $\mathrm{P})$. Quantification of $(\mathrm{M}-\mathrm{P})$ is shown $(\mathrm{Q})($ Control $+\operatorname{shCon}, \mathrm{n}=7$; Neurog $2+\operatorname{shEed}, \mathrm{n}=5)$. $(\mathrm{R}-$ W) Experiments were performed as in (A) and (D) with transfection of the indicated plasmids. The asterisk in (S) shows mis-located cells that are positive for Foxp2 (for Foxp2, shCon + 
Control, $\mathrm{n}=4 ; \operatorname{shRing} 1 A+$ Control, $\mathrm{n}=4 ; \operatorname{shCon}+\operatorname{Neurog} 2, \mathrm{n}=4 ; \operatorname{shRing} 1 A+$ Neurog $2, \mathrm{n}=$

4 | for Zfpm2, shCon + Control, $\mathrm{n}=4 ; \operatorname{shRing} 1 A+$ Control, $\mathrm{n}=3 ; \operatorname{shCon}+\operatorname{Neurog} 2, \mathrm{n}=4$; shRing $1 A+$ Neurog2, $\mathrm{n}=3$ ). Scale bars: $50 \mu \mathrm{m}(\mathrm{A}, \mathrm{D}, \mathrm{G}$, and $\mathrm{R}) ; 500 \mu \mathrm{m}(\mathrm{M})$. Data are represented as means $\pm \mathrm{SEM} ;{ }^{*} p<0.05 ; * * p<0.001 ; * * * p<0.0001$ from two-tailed unpaired Student's $t$-test with Welch's correction.

Figure 6. Involvement of Foxp2 in cortico-thalamic neuron specification and temporal regulation of its promoter by the PRC machinery

(A) mRNA levels of Fezf2 and Foxp2. Samples were collected by FACS from E17.5 cortices that had been electroporated at E13.5 with EGFP and control or Neurog2 expression vector (Control, $\mathrm{n}=6$; Neurog2, $\mathrm{n}=4$ ). (B and C) Immunostaining for Foxp2 in E18.5 cortices after in utero electroporation at E13.5 of EGFP and Fezf2 with or without shEed. (D-G) Immunostaining for Zfpm2 in E18.5 cortices after in utero electroporation at E12.5 of EGFP and the indicated plasmids. Quantification is shown in $(\mathrm{G})(\operatorname{shCon}+$ Control, $\mathrm{n}=4 ; \operatorname{sh}$ Foxp $2+$ Control, $\mathrm{n}=4 ; \operatorname{shCon}+$ Neurog $2, \mathrm{n}=3 ; \operatorname{shFoxp} 2+$ Neurog $2, \mathrm{n}=4)$. (H and I) Immunostaining for Zfpm2 in E18.5 cortices after in utero electroporation at E13.5 of EGFP and the indicated plasmids. Quantification is shown in (I) $([$ Neurog $2+\operatorname{shEed}]+\operatorname{shCon}, \mathrm{n}=4 ;[$ Neurog $2+\operatorname{shEed}]$ $+\operatorname{shFoxp} 2, \mathrm{n}=4) .(\mathrm{J}-\mathrm{N})$ Immunostaining for EGFP in P3 brains after in utero electroporation at E13.5 of EGFP and the indicated plasmids. A representative image is shown in each condition. Dotted circles at a posterior level indicate the dorsal thalamus ( $\mathrm{K}$ and $\mathrm{M})$. Quantification of $(\mathrm{J}-\mathrm{M})$ is shown $(\mathrm{N})([$ Neurog $2+\operatorname{shEed}]+\operatorname{shCon}, \mathrm{n}=4 ;[$ Neurog $2+\operatorname{shEed}]$ $+\operatorname{shFoxp} 2, \mathrm{n}=4)$. (O and $\mathrm{P})$ ChIP-qPCR analysis with $\mathrm{H} 3 \mathrm{~K} 27 \mathrm{me} 3$ antibody $(\mathrm{O})$ or H2AK119ub antibody (P) using chromatin prepared from E12.5 and E14.5 cortices. The 
amount of promoter regions of Amyl (as a control) and Foxp2 genes in the immunoprecipitated chromatin was detected by qPCR. The percentages of input chromatin were quantified in duplicate from three independent chromatin samples of different litters. (Q-S) Immunostaining for Bc111b in E18.5 cortices after in utero electroporation at E13.5 of EGFP and control or Foxp2 expression vector. The percentage of Bcl11b-positive cells among EGFP-positive cells was quantified (S) (Control, $\mathrm{n}=4 ;$ Foxp2, $\mathrm{n}=3)$. $(\mathrm{T}-\mathrm{V})$ Axon trajectories in $\mathrm{P} 3$ brains from the neurons that had been electroporated with EGFP and Foxp2 vector at E13.5. A representative image is shown. Dotted circles at a posterior level indicate the dorsal thalamus. Quantification is shown (V) (Control, $\mathrm{n}=7$; Foxp2, $\mathrm{n}=4)$. Scale bars: $50 \mu \mathrm{m}(\mathrm{B}, \mathrm{D}, \mathrm{H}$, and Q); $500 \mu \mathrm{m}(\mathrm{J}$ and $\mathrm{T})$. Data are represented as means $\pm \mathrm{SEM} ;{ }^{*} p<0.05 ; * * p<0.005 ; * * * p<0.0001$ from two-tailed unpaired Student's $t$-test with Welch's correction.

\section{Figure 7. Schematic representation of the regulatory cascade for cortico-thalamic neuron} specification and of its temporal control

(A) Neurog $1 / 2$ specify the CTN fate in layer 6 by inducting Fezf 2 expression via direct binding to its enhancer. Neurog1/2 may also activate other targets including Nhlh1. Fezf2, in turn, promotes Foxp2 expression, presumably by directly activating its promoter. This gene cascade contributes to the establishment of the CTN fate, which antagonizes another major fate of layer 6 neurons, cortico-cortical neurons. (B) In E12.5 NPCs, the gene cascade described in (A) is active. However, this cascade is blocked in NPCs after E12.5 by two independent mechanisms. Neurog1/2 transcriptional activity decreases, presumably because of reduced recruitment of coactivators including p300. CTN fate determinant genes including Foxp2 become inaccessible because of repressive modifications catalysed by PRC1, which ubiquitinates K119 of Histone 
bioRxiv preprint doi: https://doi.org/10.1101/431684; this version posted October 1, 2018. The copyright holder for this preprint (which was not certified by peer review) is the author/funder. All rights reserved. No reuse allowed without permission.

$\mathrm{H} 2 \mathrm{~A}$. 


\section{Figure 1}

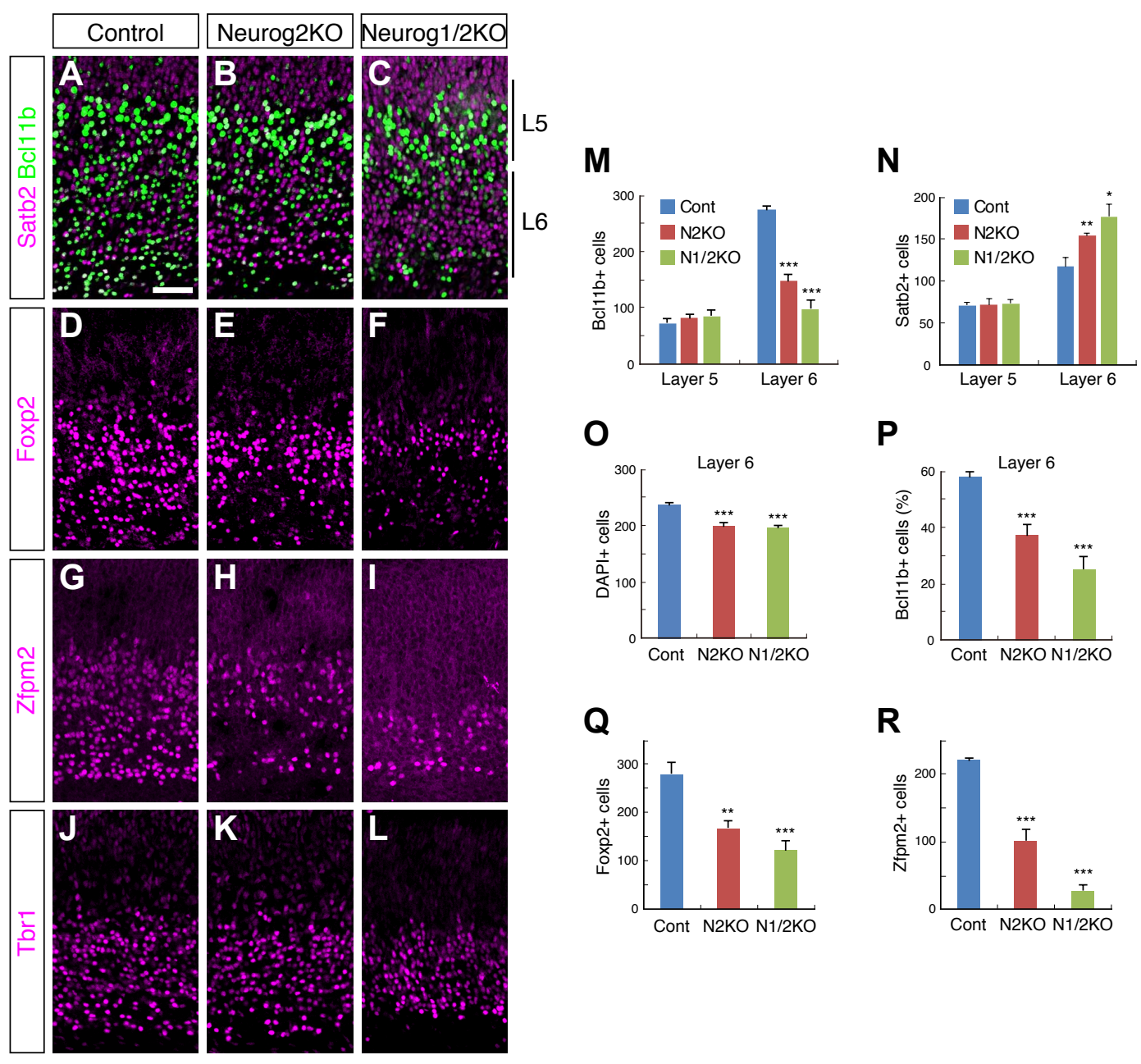




\section{Figure 2}

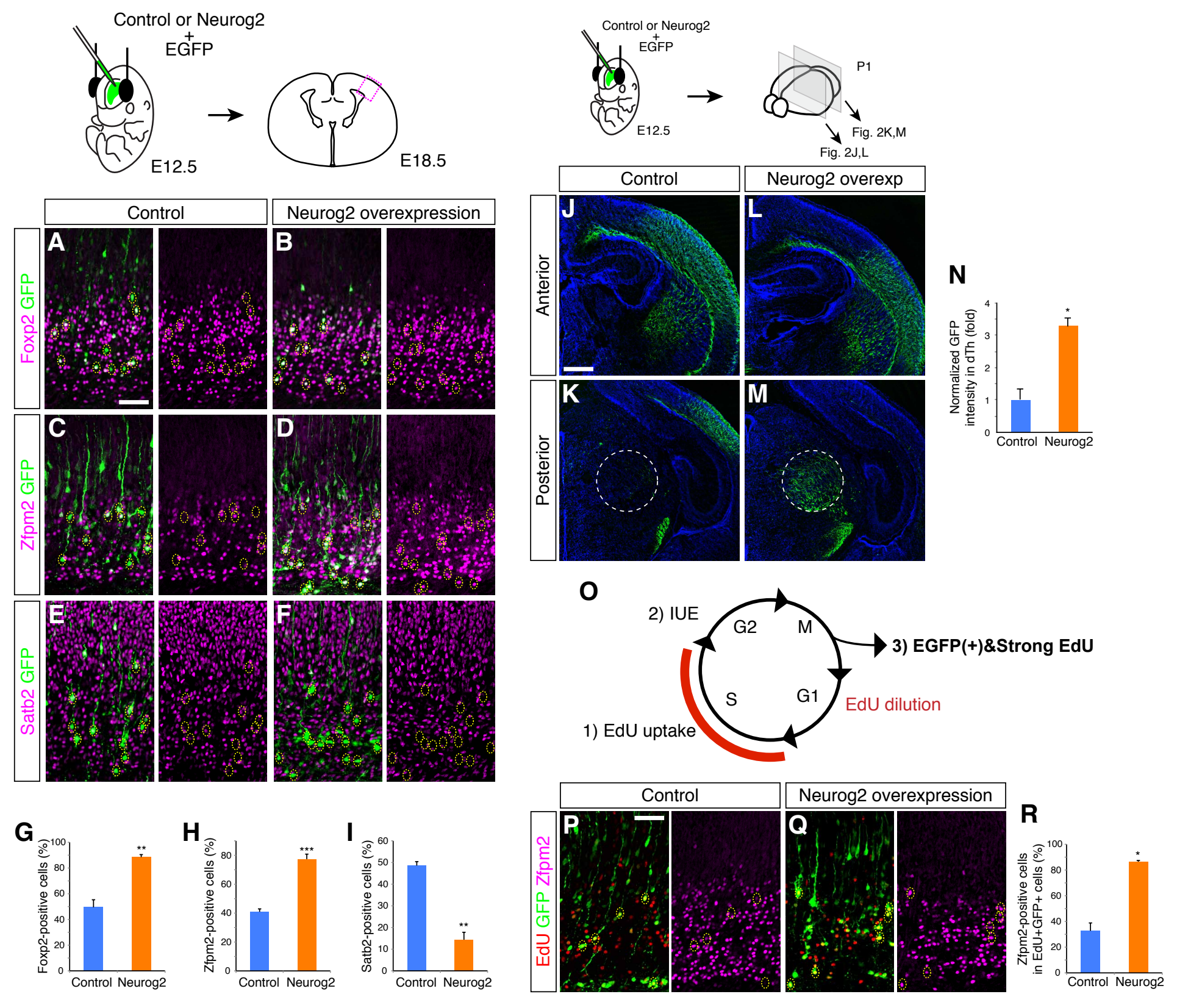


bioRxiv preprint doi: https://doi.org/10.1101/431684; this version posted October 1,2018 . The copyright holder for this preprint (which was not certified by peer review) is the author/funder. All rights reserved. No reuse allowed without permission.

\section{Figure 3}

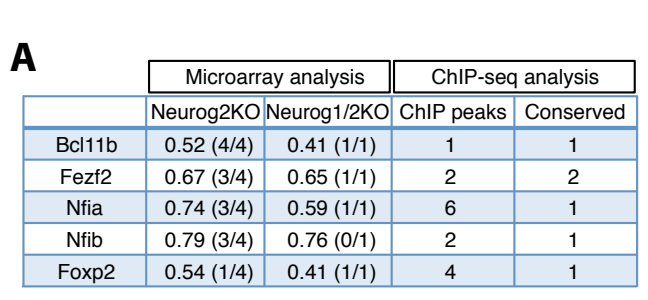

B

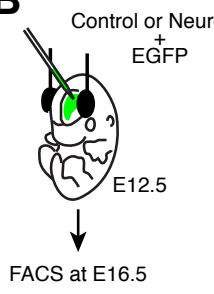

C

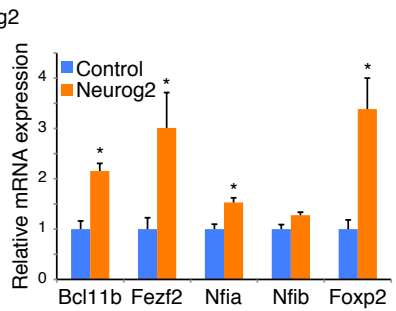

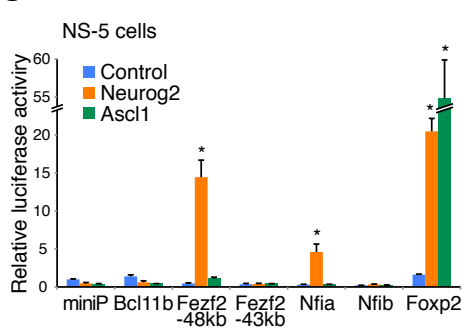

D

Fezf2-48kb Neurog2-binding region

GCGACAGCATAAGAAATAAACACTTGTGGTTCATTCGAAGGGGGAATGAAATTGAATAAGCTCTCAGCACTTGAGGGGCACCACAAGCGCCTGAATG $10 \quad 20 \mathrm{E} 130$

GGGCAAGCCTCCTAAGTTGAATGGAGTGTCTTTTGGGACCAATTTGTCTCTTTTCACTCTTTATTCTTCTCTTTTTGCTGACAGATGCACAAAGGGGAA

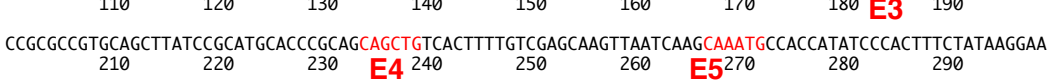

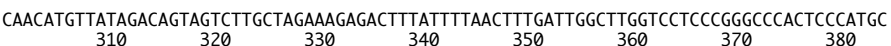

E

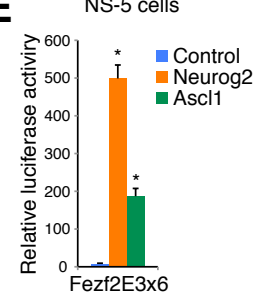

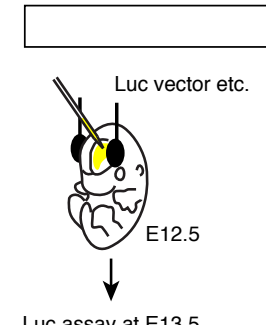

Luc assay at E13.5
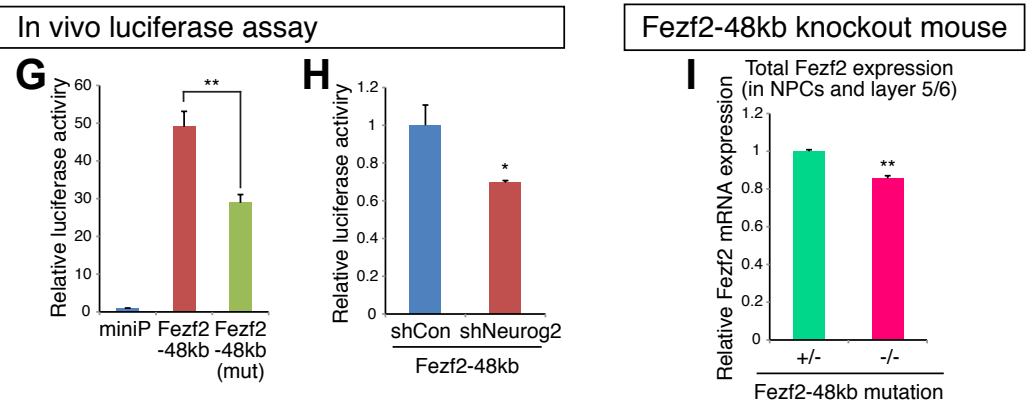

F NS-5 cells

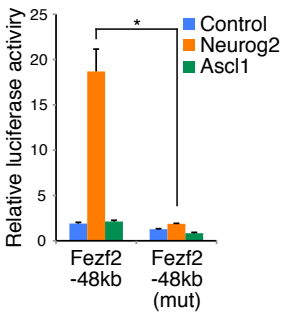

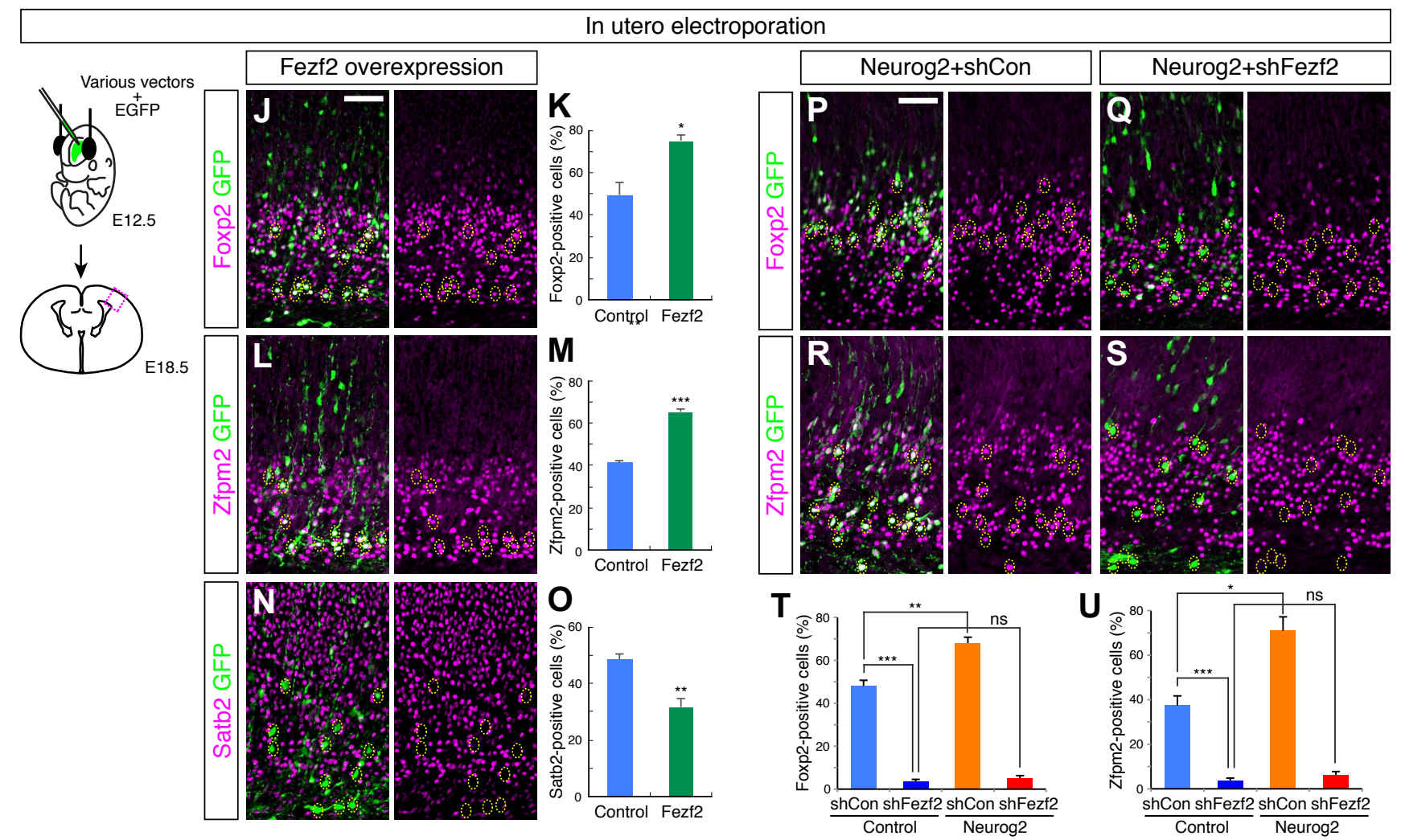


Figure 4 not certified by peer review) is the author/funder. All rights reserved. No reuse allowed without permission.

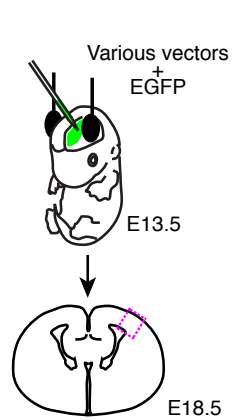

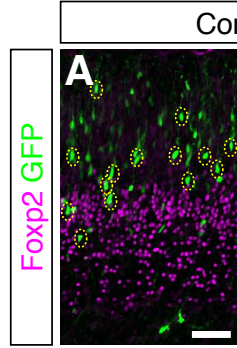

\section{Control}
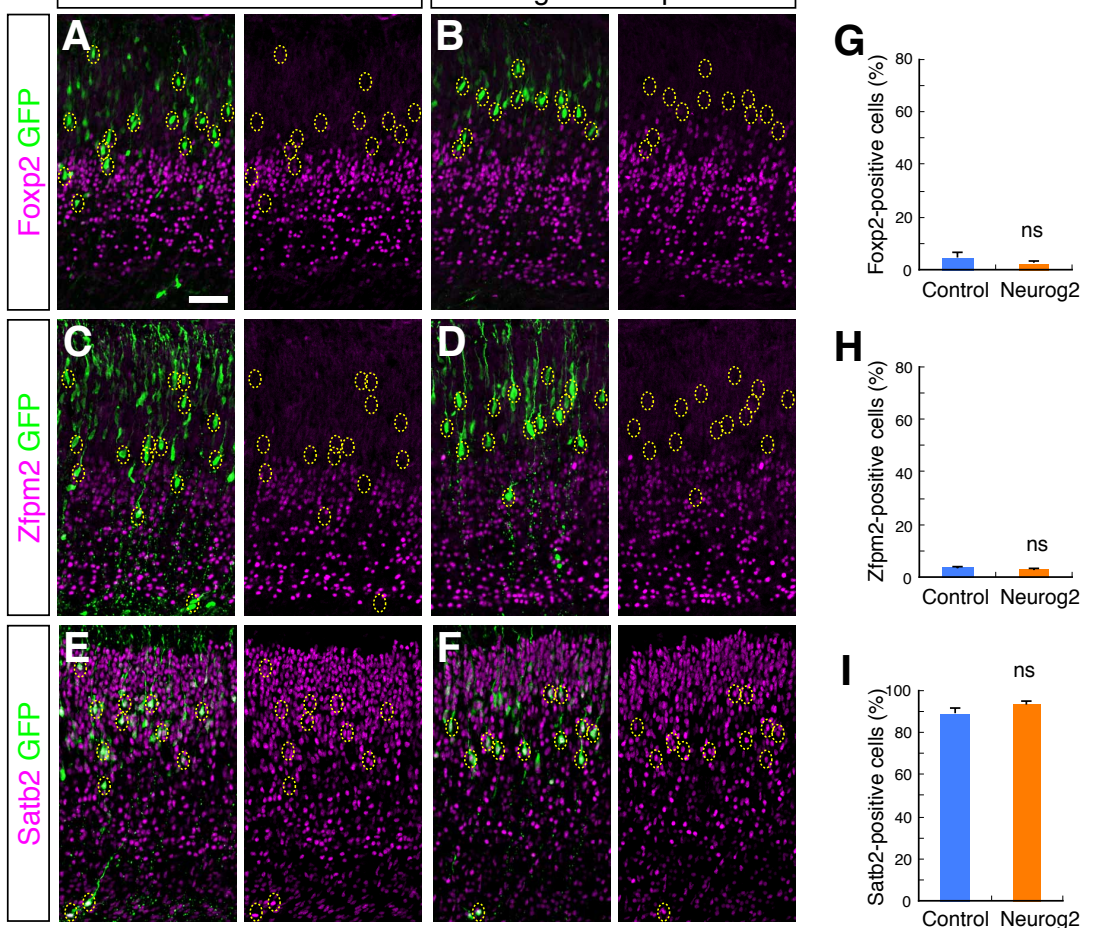

J

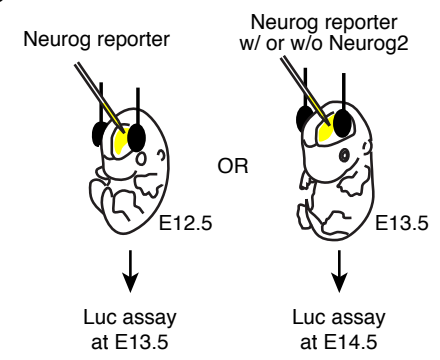

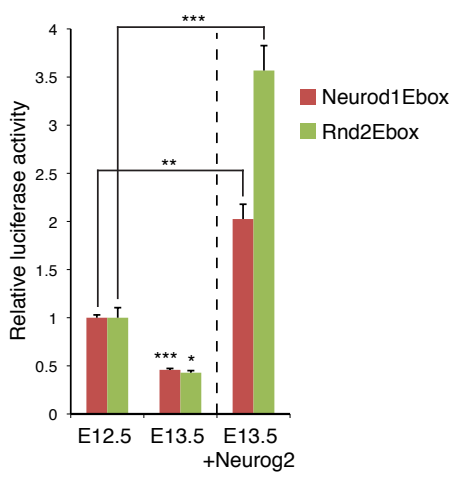


Figure 5

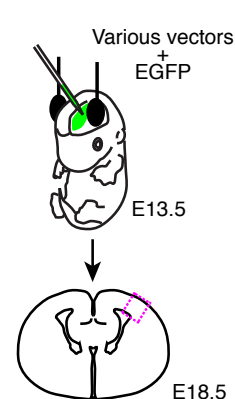

Fig. 5A-L,R-W

OR

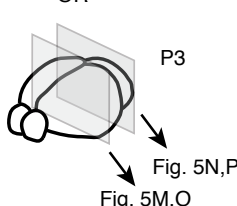

Fig. $5 \mathrm{M}, \mathrm{O}$
E13.5 IUE -> E18.5
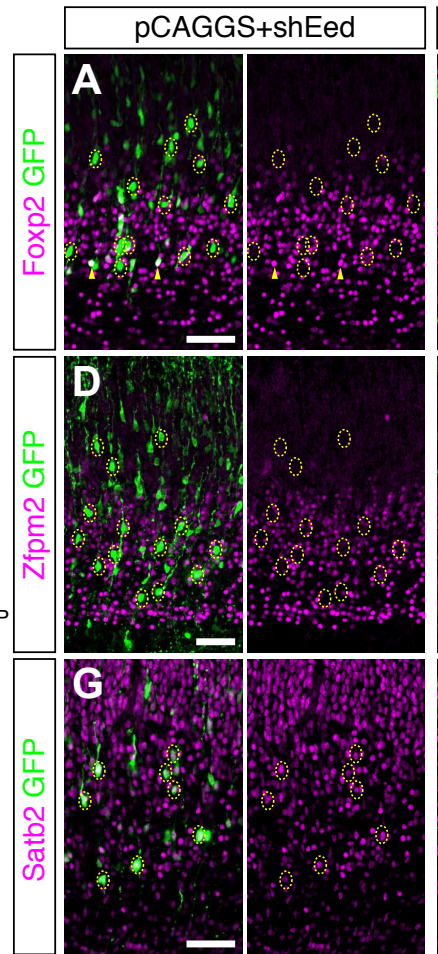

E13.5 IUE $\rightarrow$ P3

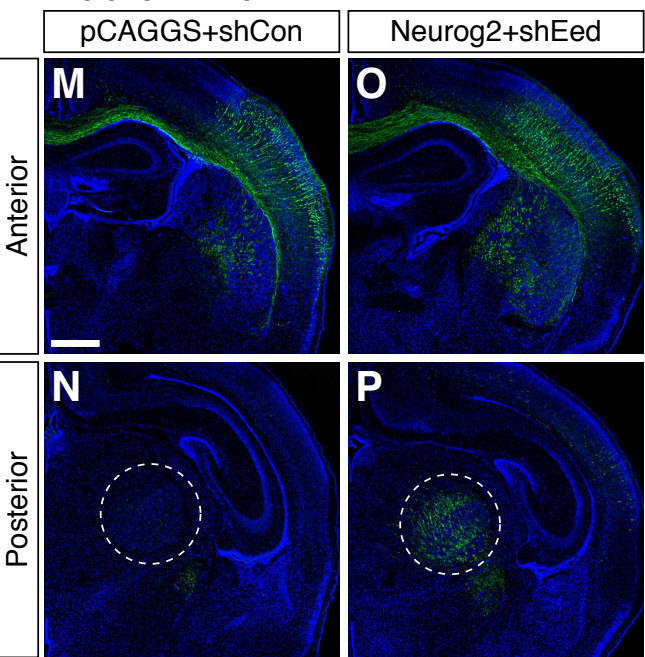

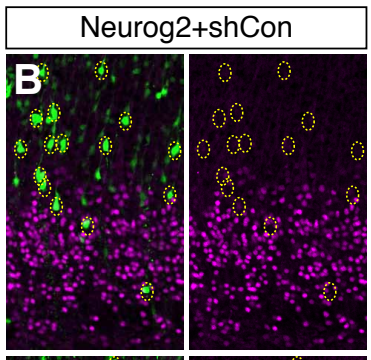
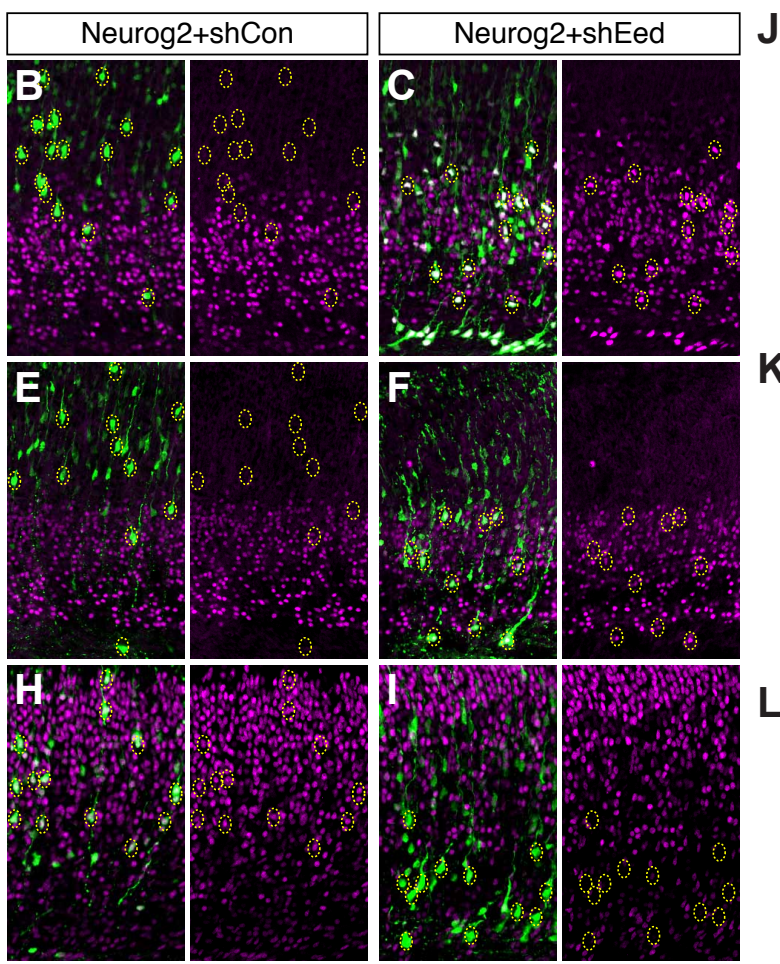

K
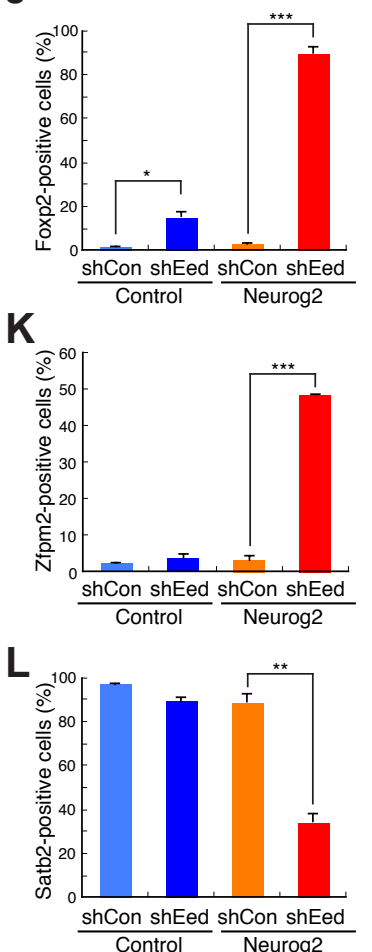

E13.5 IUE -> E18.5

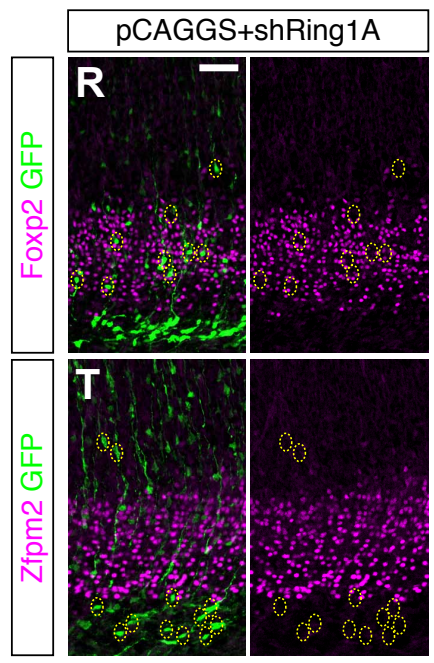

Q

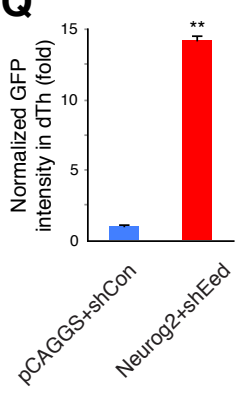

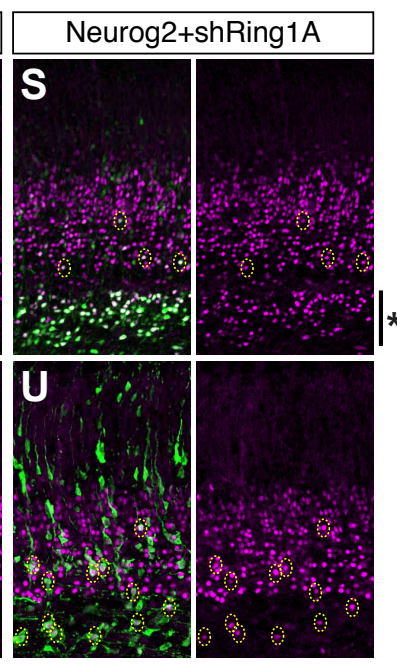
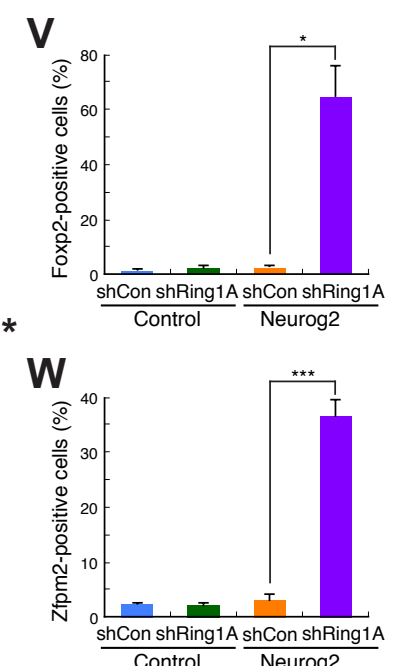
bioRxiv preprint doi: https://doi.org/10.1101/431684; this version posted October 1, 2018. The copyright holder for this preprint (which was Figure 6 not certified by peer review) is the author/funder. All rights reserved. No reuse allowed without permission.
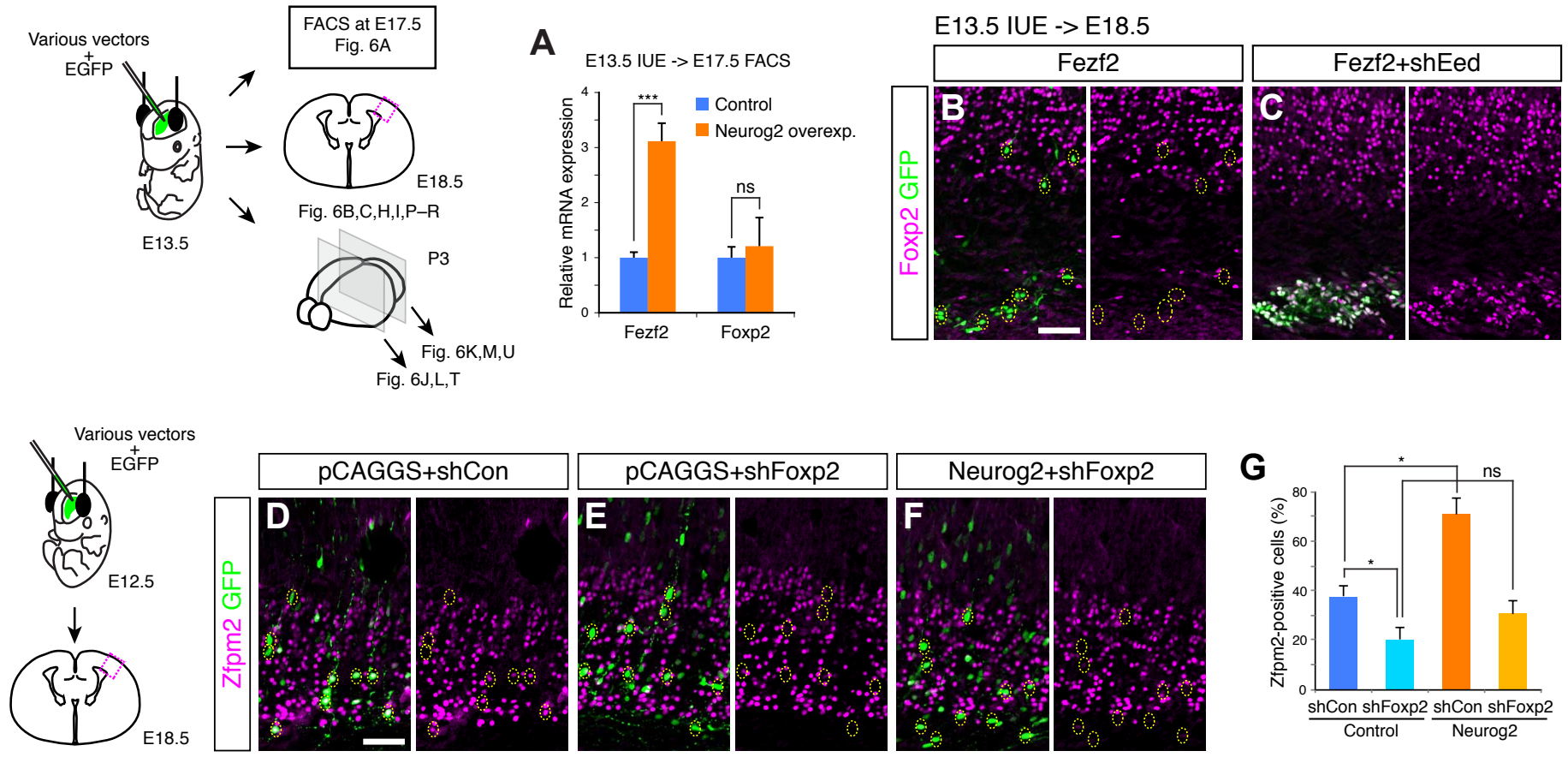

H E13.5 IUE -> E18.5
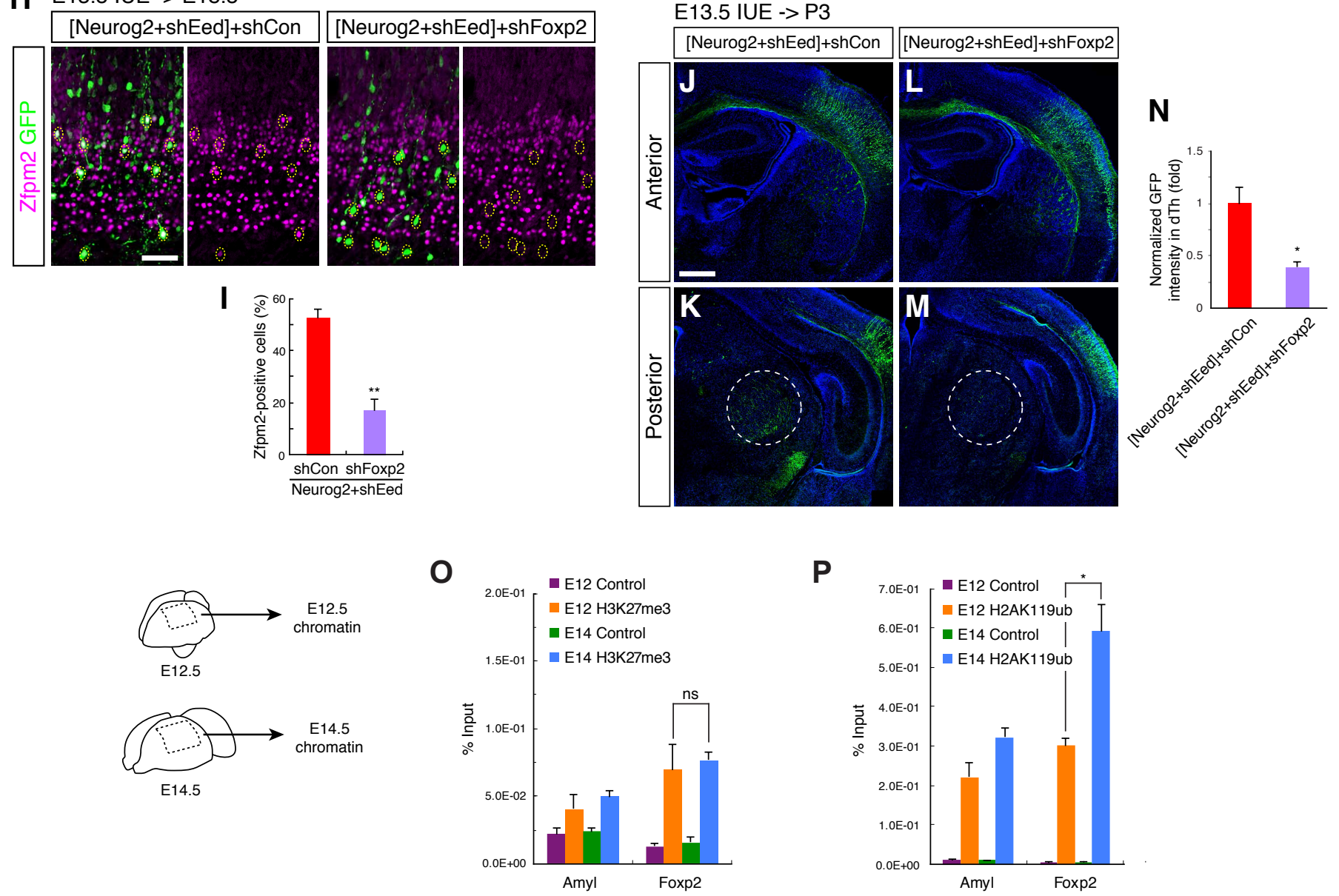

E13.5 IUE -> E18.5

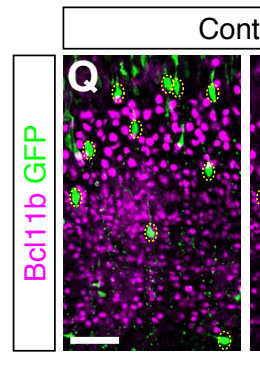

Control

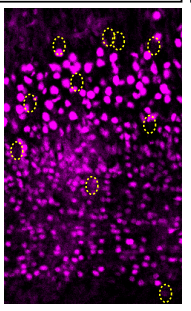

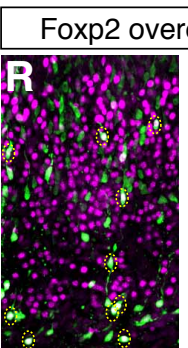
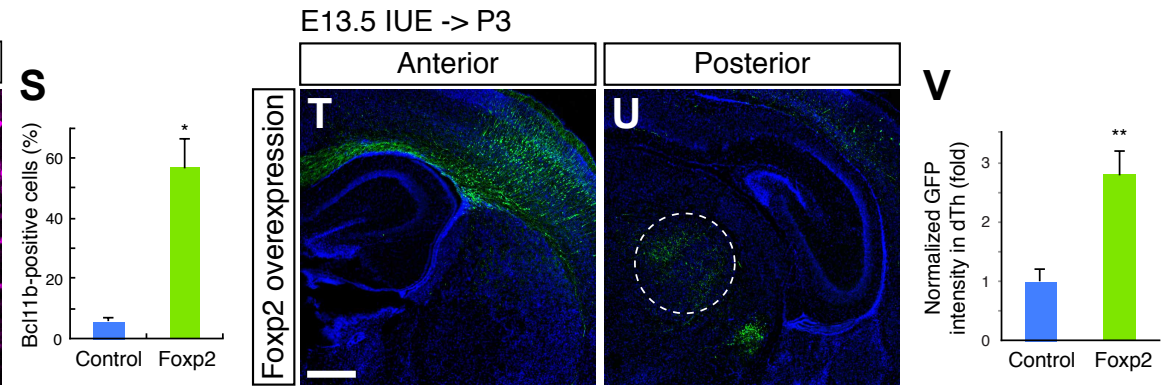
Figure 7
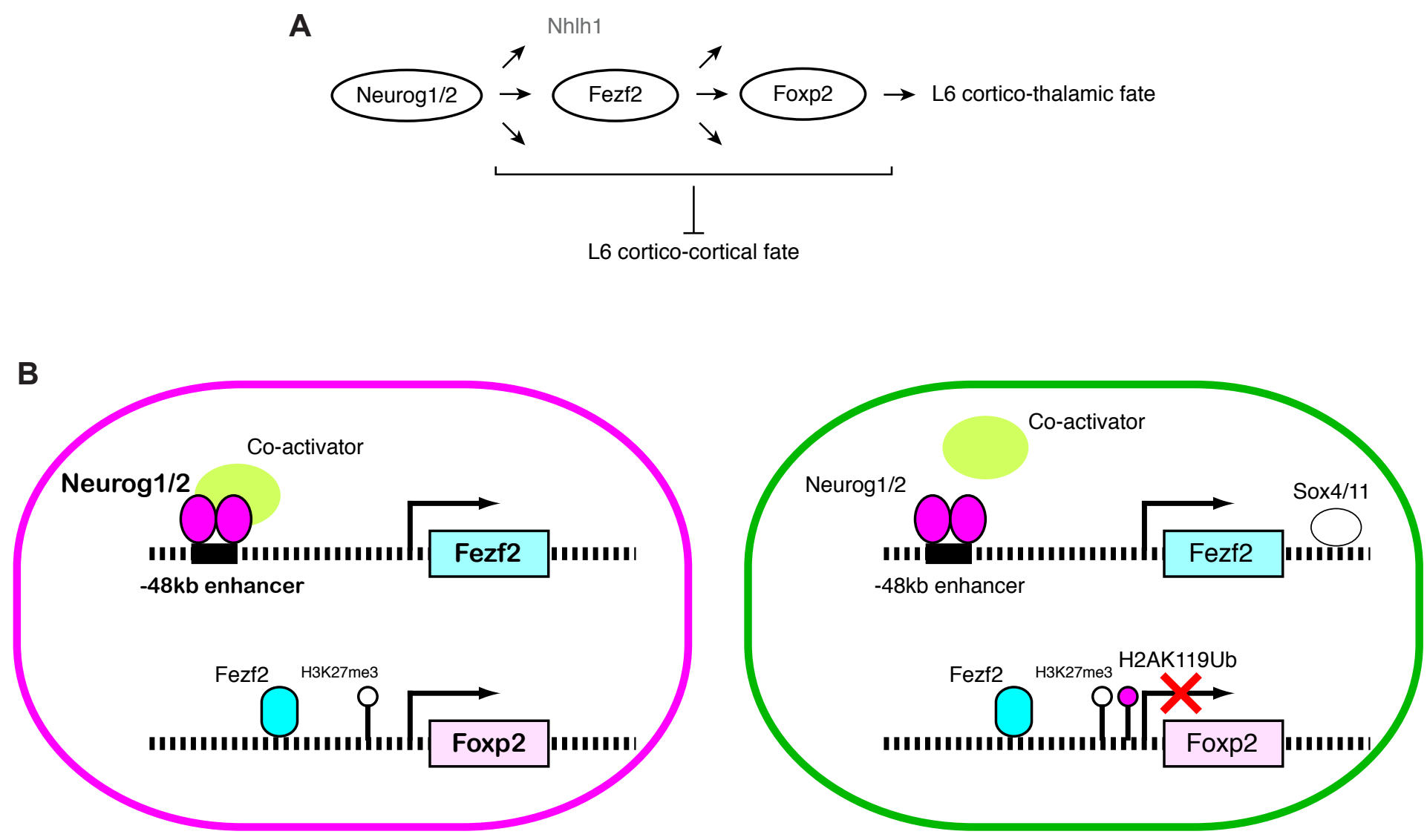

E12.5 NPCs

E13.5 NPCs 